\title{
High leverage points and vertical outliers resistant model selection in regression
}

\author{
Kundalik S. Shende*(D), Dattatraya N. Kashid(D) \\ Department of Statistics, Shivaji University, Kolhapur, India (MS)
}

\begin{abstract}
It is necessary to consider only relevant predictor variables for prediction purpose because irrelevant predictors in the regression model will tend to misleading inference. There are so many model selection methods available in the literature; among these, some methods are resistant to vertical outliers, but still, the problem of the presence of vertical outliers and leverage points is not well studied. In this article, we have modified the $S_{p}$ statistic using the generalized M-estimator for robust model selection in the presence of vertical outliers and high leverage points. The proposed model selection criterion selects only relevant predictor variables by probability one for a large sample size. We found the equivalence of this criterion and the existing $C_{p}$ and $S_{p}$ criteria. The superiority of a proposed criterion is demonstrated using simulated and real data.
\end{abstract}

Mathematics Subject Classification (2020). 62J05, 62F35

Keywords. GM-estimator, adaptive $S_{p}$ statistic, $S_{p}$ statistic, $C_{p}$ statistic, consistency, robust model selection

\section{Introduction}

The prime intention behind the use of a regression model is to predict the unknown response variable for the given values of predictor variables. The prediction of the response variable depends on the predictor variables in the model. The relevant predictor variables in the model give accurate predictions. Model selection methods consider only relevant predictor variables. The general form of a multiple linear regression model is

$$
y=X \beta+\varepsilon,
$$

where $y$ is a vector of $n$ observations on a response variable, $\beta=\left(\beta_{0}, \beta_{1}, \beta_{2}, \ldots, \beta_{k-1}\right)^{\prime}$ is a vector of unknown $k$ regression coefficients, $X$ is a matrix of size $n \times k$ of observations on $(k-1)$ predictor variables $X_{1}, X_{2}, X_{3}, \ldots, X_{(k-1)}$ with 1 's in the first column and $\varepsilon$ is a vector of errors with $E(\varepsilon)=0$ and $\operatorname{Var}(\varepsilon)=\sigma^{2} I_{n}$. The full model (1.1) can be written as

$$
y=X_{1} \beta_{1}+X_{2} \beta_{2}+\varepsilon,
$$

where $X$ and $\beta$ are partitioned as $X=\left[X_{1}: X_{2}\right]$ and $\beta^{\prime}=\left[\beta_{1}^{\prime}: \beta_{2}^{\prime}\right] . X_{1}$ is $n \times p$ matrix of observations on $(p-1)$ predictor variables with 1 's in the first column and $\beta_{1}$ is a vector

\footnotetext{
*Corresponding Author.

Email addresses: shendeks.stat99@gmail.com (K.S. Shende), dnk_stats@unishivaji.ac.in (D.N. Kashid)

Received: 18.12.2020; Accepted: 16.07.2021
} 
of corresponding unknown regression coefficients. Similarly, $X_{2}$ is $n \times(k-p)$ matrix of observations on remaining predictor variables and $\beta_{2}$ is a vector of corresponding unknown regression coefficients. Now, consider a submodel based on $p-1(p<k)$ predictor variables,

$$
y=X_{1} \beta_{1}+\varepsilon .
$$

We can select an appropriate model by testing the null hypothesis $H_{0}: \beta_{2}=0$ or using criterion function available in the literature. Many model selection criteria have been proposed based on the classical least squares (LS) estimator. Among these, Mallows's $C_{p}$ $[22]$ is a well-known model selection technique. It is defined as

$$
C_{p}=\frac{R S S_{p}}{\sigma^{2}}-(n-2 p),
$$

where $R S S_{p}$ is residual sum of squares of the submodel which has $(p-1)$ predictor variables. $\sigma^{2}$ is an unknown error variance, and it can be replaced by its suitable estimate like residual mean squares of the full model. The LS estimator is used to calculate $R S S_{p}$ and residual mean squares in Mallows's $C_{p}$. It is well-known that the LS estimator is optimal and strongly efficient when assumptions of regression are satisfied. However, an outlier in the data destroys the LS estimator, and consequently, it is also affecting on Mallows's $C_{p}$ and other LS estimator based methods.

Several robust estimators alternative to the LS estimator have been proposed in the literature to overcome this issue. The M-estimator [17] is prominent broad class of robust estimators, and it reduces the effect of outliers by assigning low weights to the outliers. Researchers have suggested robust model selection methods based on this M-estimator. Rao et al. [25] have reviewed many non-robust and robust model selection criteria. Ronchetti and Staudte [28] have proposed a robust version of Mallows's $C_{p}$ based on the M-estimator. It is defined as

$$
R C_{p}=\frac{W_{p}}{\hat{\sigma}^{2}}-\left(U_{p}-V_{p}\right),
$$

where $W_{p}$ is the weighted residual sum of squares of the submodel, $U_{p}$ and $V_{p}$ are constants depends on weight function and the number of parameters in the corresponding model, and $\hat{\sigma}^{2}$ is a robust and consistent estimate of error variance based on a full model. The $R C_{p}$ criterion selects the model whose $R C_{p}$ value is close to $V_{p}$. Further, Kashid and Kulkarni [18] proposed a more simple robust model selection $S_{p}$ criterion based on the M-estimator. It is defined as

$$
S_{p}=\frac{\left\|\hat{y}_{k}-\hat{y}_{p}\right\|^{2}}{\sigma^{2}}-(k-2 p),
$$

where $\|\cdot\|$ represents $L_{2}$ norm, $\hat{y}_{k}$ and $\hat{y}_{p}$ are vector of predicted values of $y$ based on full model and submodel respectively. The M-estimator is used to calculate these predicted values. An unknown error variance $\sigma^{2}$ is replaced by its suitable robust estimate. The $S_{p}$ criterion selects the model whose $S_{p}$ value is close to the number of unknown parameters $(p)$ in the submodel.

Kim and Hwang [19] proposed a method based on Mallows's $C_{p}$ called as $C_{p}(d)$ by deleting $d$ outlying observations to select relevant predictor variables. A robust version of Akaike information criterion (RAIC) [27] and robust version of Bayesian information criterion (RBIC) [21] are also available in the literature to select a model in presence of outliers. Tharmaratnam and Claeskens [32] have compared the classical AIC criterion with a robust version of AIC based on different robust estimators in the presence of outliers. André et al. [2], Croux and Dehon [10], Maronna et al. [23], Renaud and Victoria-Feser [26] proposed different robust coefficient of determination based on robust estimators to identify appropriate predictor variables and assess the quality of the model. All the aforesaid robust model selection methods are robust to vertical outliers. 
Generally, three types of outliers namely, vertical outliers, bad leverage points and good leverage points are considered in the regression analysis. Vertical outliers and bad leverage points are outlying only in Y-space and X-space respectively, and these types of outliers are located far away from the regression line. Good leverage points are outlying in both space, and they are found near to the regression line. The vertical outliers and bad leverage points are significantly affecting the estimated regression parameters, while good leverage points are not affecting the estimated regression parameters [11]. All these outlying points can be identified by using methods available in the literature $[1,3,4,6,8,12,29,30]$.

The M-estimator is an one of the widely used robust estimator in regression, but it fails to account the high leverage point in the parameter estimation [33] and consequently in model selection methods. Almost all the above-cited methods are introduced to curb the effect of vertical outliers but are not performing well in the presence of leverage points. In response to this problem, Mallows and Schweppe [20,33] have suggested a generalized M (GM) estimator as an alternative to classical M-estimator. The GM-estimator $\hat{\beta}$ of $\beta$ for a linear regression model is the solution of an equation,

$$
\sum_{i=1}^{n} \eta\left(X_{i}, \frac{y_{i}-X_{i}^{\prime} \beta}{\sigma}\right) X_{i}=0 .
$$

In general, the function $\eta$ can be represented as

$$
\eta(X, r)=\omega(X) \psi(r \nu(X)),
$$

where $\omega$ and $\nu$ are weight functions such that, $\omega: \mathbb{R}^{k} \rightarrow \mathbb{R}^{+}, \nu: \mathbb{R}^{k} \rightarrow \mathbb{R}^{+}, \psi: \mathbb{R} \rightarrow \mathbb{R}$, and $r$ is standardized residual [13]. Mallows and Schweppe [20,33] have recommended the function $\eta$ choosing $\nu(X)=1$ and $\nu(X)=\frac{1}{\omega(X)}$ weight functions respectively. Thus, the function $\eta$ for Schweppe type estimator is defined as

$$
\eta(X, r)=\omega(X) \psi\left(\frac{r}{\omega(X)}\right)
$$

where $\psi(\cdot)$ is odd, bounded, uniformly continuous, non-decreasing and $\psi(u)>0$ for $u>0$ [24]. Hill [14] pointed out that, Mallows and Schweppe estimators are more efficient than several other estimators with Schweppe's method having an advantage. The main advantage of GM-estimator with the Schweppe's weighting scheme is that it assigns a weight to the high leverage point considering its distance from the regression line (i.e. assigns low weight to the leverage point if it has high residual) $[9,13]$. The diagonal values $\left(h_{i i}\right)$ of the hat matrix $H=X\left(X^{\prime} X\right)^{-1} X^{\prime}$ are used to identify leverage points. Generally, $h_{i i}>2 k / n$ (or $3 k / n$ ) indicates that the corresponding observation is a high leverage point $[6,20,29]$. It is necessary to assign a low weight to this observation in the parameter estimation to reduce the effect of leverage point on regression parameters. This can be achieved by the weight function $\omega\left(X_{i}\right)=\sqrt{1-h_{i i}}$ because $\omega\left(X_{i}\right)<\omega\left(X_{j}\right)$ for $h_{i i}>h_{j j}, i \neq j=1,2,, n$. Therefore, in this article we propose a model selection criterion based on the GM-estimator with Schweppe's weight function, $\nu\left(X_{i}\right)=\frac{1}{\omega\left(X_{i}\right)}$ and $\omega\left(X_{i}\right)=\sqrt{1-h_{i i}}, i=1,2, \ldots, n$. Equation (1.7) can be solved using iterative method, and at convergence the GM-estimator is given as,

$$
\hat{\beta}=X\left(X^{\prime} W X\right)^{-1} X^{\prime} W y,
$$

where the final diagonal weight matrix $W, W_{i i}=\frac{\eta\left(X_{i}, r_{i}\right)}{r_{i}}, i=1,2, \ldots, n$ is obtained by using $\omega(X)$ and $\psi(\cdot)$ function.

The remaining article has organized as follows: Section 2 explains the problem of the existence of a vertical outlier and a leverage point in the data and evaluates the performance of existing methods. In Section 3, the new criterion is proposed based on the GM-estimator to combat the simultaneous occurrence of vertical outliers and high leverage points. Also, the consistency property of the proposed method has established. An 
extensive simulation study is carried out in Section 4 to illustrate the performance of the proposed criterion and compared with existing methods through the real data. The article ends with a discussion given in Section 5 .

\section{The problem}

In this section, we consider an example to illustrate the effect of the simultaneous occurrence of a vertical outlier and a leverage point in the data. Consider the regression model

$$
y_{i}=5+3.5 X_{i 1}+6 X_{i 2}+0 X_{i 3}+0 X_{i 4}+\varepsilon_{i},
$$

where $\varepsilon_{i}, i=1,2, \ldots, 50$ are independent and identical errors generated from a standard normal distribution. The predictor variables $X_{j}(\mathrm{j}=1,2,3,4)$ are generated from standard uniform distribution and using the above model, we generate the response $y$. A vertical outlier has introduced in the data multiplying by three to a response variable corresponding to the highest absolute residual. The leverage point has introduced in the data multiplying by three to a row of the $X$ matrix (excluding 1's column) corresponding to the highest leverage $\left(\max \left(h_{i i}\right)\right)$. The plot of Modified Generalized Studentized Residuals (MGti) versus the Diagnostic Robust Generalized Potential (DRGP) is used to identify the outliers in the data [1].

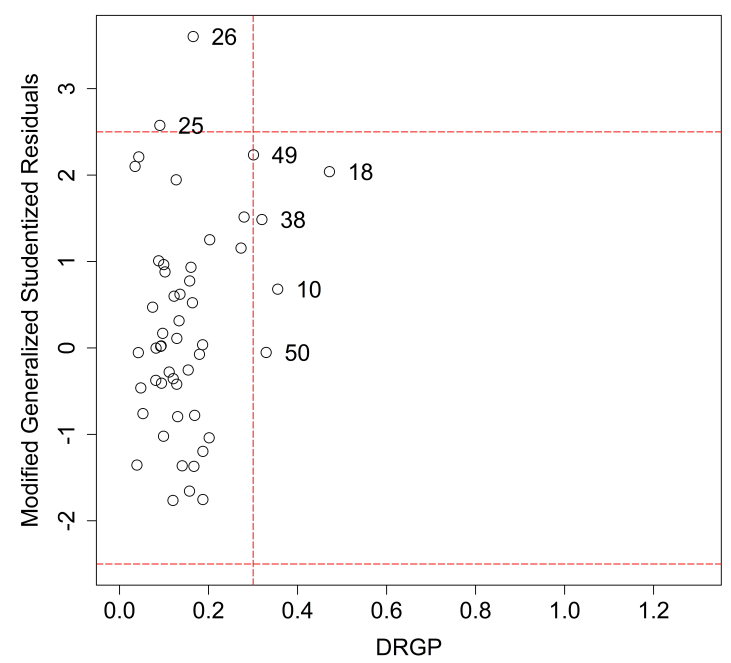

(a)

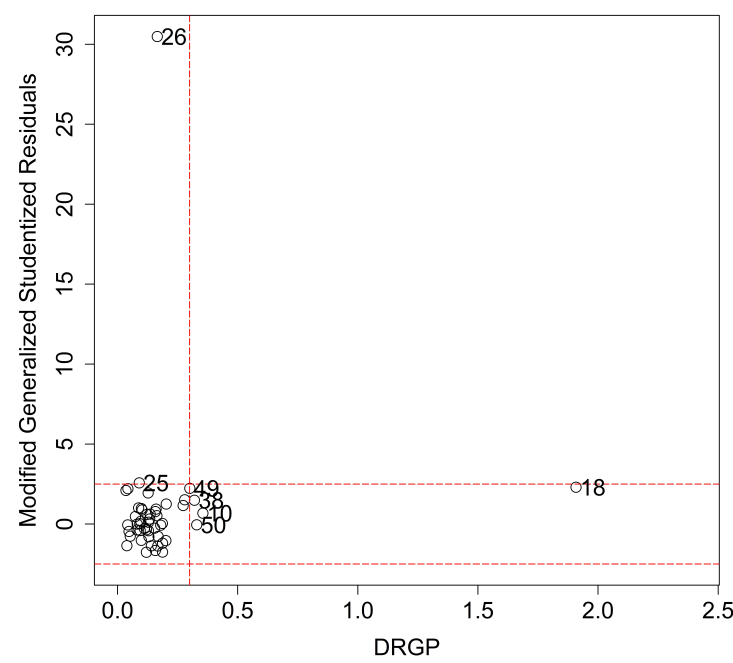

(b)

Figure 1. (a) MGt-DRGP plot for original data, (b) MGt-DRGP plot in the presence of a vertical outlier and a leverage point.

In the simulated data from the model under consideration, $26^{\text {th }}$ observation has the largest absolute standardized residual and MGt value, and $18^{\text {th }}$ observation has the largest leverage and DRGP value. To make these observations as a vertical outlier and a high leverage point multiplying by three to $y_{26}$ and $18^{\text {th }}$ row of an $X$ matrix (excluding 1's column) respectively. Thus, the modified data has influential vertical outlier as well as mild bad leverage point (Figure 1 (b)).

We have evaluated the performance of non-robust and robust model selection methods using the above simulated data. We compute $C_{p}, S_{p}, R C_{p}$ and $S_{p}$ (based on GM-estimator) for all possible submodels using simulated data and compare by plotting values of these statistics (see Figure 2). The classical $C_{p}$ chooses different subset of predictor variables 
$\left(X_{1}, X_{2}, X_{3}\right),\left(X_{1}, X_{2}, X_{4}\right)$ and $\left(X_{1}, X_{2}, X_{3}, X_{4}\right)$ according to 'close to $p$ ' criterion. Hence, $C_{p}$ select overfitted model means a model having all relevant predictor variables and at least one irrelevant predictor variable. However, the value of the $C_{p}$ statistic corresponds to correct subset $X_{1}, X_{2}$ is very small as compared to $p$ and this incline to select overfitted model. This indicates that, $C_{p}$ statistic is sensitive to vertical outliers and leverage points. The $R C_{p}$ and $S_{p}$ are M-estimator based robust model selection criteria choose same subset of predictor variables $X_{1}, X_{2}, X_{3}$. This model has extra irrelevant variable $X_{3}$ and is overfitted.

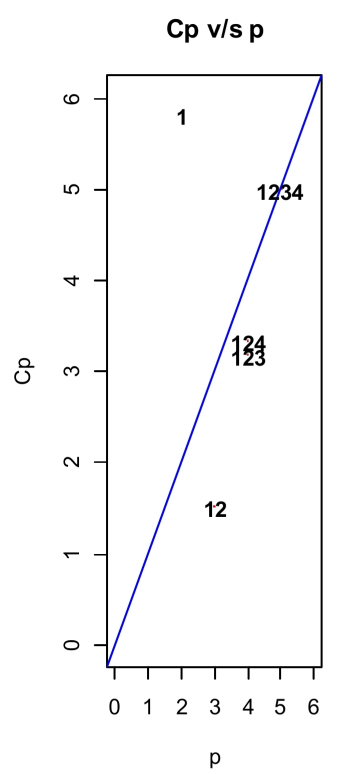

(a)

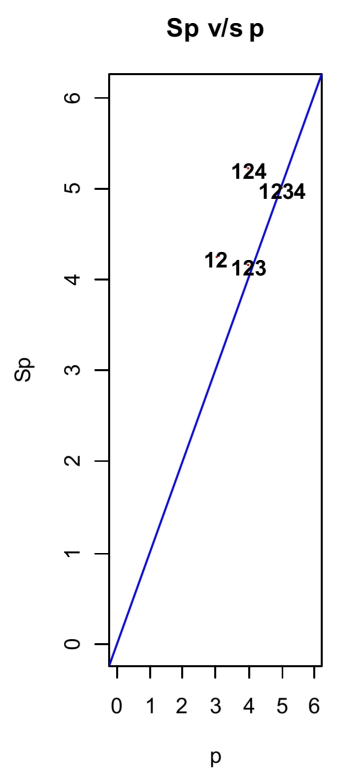

(b)

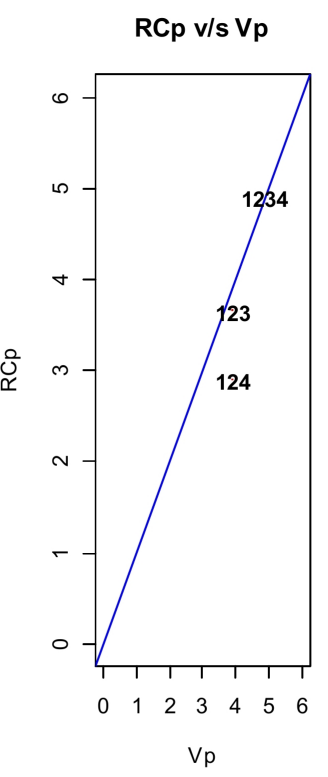

(c)

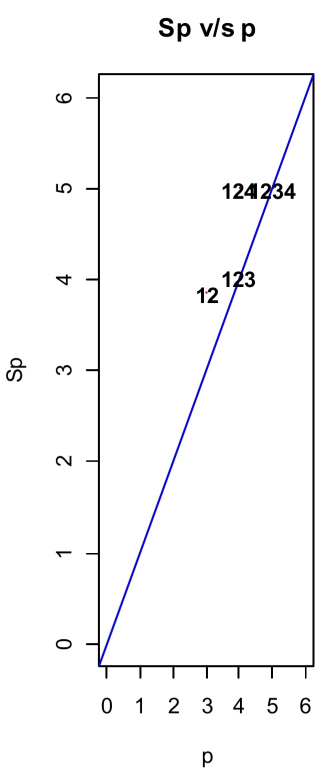

(d)

Figure 2. a) $C_{p}$ versus $p$, (b) $S_{p}$ versus $p$, (c) $R C_{p}$ versus $V_{p}$, (d) $S_{p}$ (based on GM) versus $p$.

In the light of the above discussion, model selection methods based on LS estimator and M-estimator fails to select a proper model in the presence of both a vertical outlier and a high leverage point. Also, $S_{p}$ statistic based on GM-estimator fails to select the correct model for this situation. Hence, it is not enough to change the estimator only; it is also needed to modify the form of criterion. In next Section, we have proposed adaptive $S_{p}$ statistic based on GM estimator to tackle the same problem.

\section{Adaptive $S_{p}\left(A S_{p}\right)$}

Let $\hat{\beta}$ be the GM-estimator of $\beta$ and $\hat{y}_{k}$ be the vector of predicted values of $y$ based on the full model (1.1) using the GM-estimator. The vector of predicted values $\hat{y}_{k}$ is given by $\hat{y}_{k}=X \hat{\beta}=H y, H=X\left(X^{\prime} W X\right)^{-1} X^{\prime} W$ is a hat or projection matrix, $W$ is a diagonal matrix of non-negative weights. Let $\hat{\beta}_{1}$ be the GM-estimator of $\beta_{1}$ and $\hat{y}_{p}$ be the vector of predicted values of $y$ based on the submodel (1.3) using the GM-estimator. The fitted equation based on submodel is $\hat{y}_{p}=X_{1} \hat{\beta}_{1}=H_{p} y, H_{p}=X_{1}\left(X_{1}^{\prime} W_{1} X_{1}\right)^{-1} X_{1}^{\prime} W_{1}$ is a hat or projection matrix, $W_{1}$ is a diagonal matrix of non-negative weights. A good model selection criterion is one which considers the goodness of fit as well as the complexity of the model [7]. Therefore, we propose adaptive $S_{p}$ statistic $\left(A S_{p}\right)$ based on predicted values $\hat{y}_{k}, \hat{y}_{p}$ and model complexity measure $C(n, p)$. It is defined as

$$
A S_{p}=\frac{\left\|\hat{y}_{k}-\hat{y}_{p}\right\|^{2}}{\sigma^{2}}+C(n, p)
$$


where $\sigma^{2}$ is an unknown error variance which can be replaced by using suitable estimate $\hat{\sigma}=1.48 \times$ Median(largest $(\mathrm{n}-\mathrm{k}+1)$ absolute residuals of full model), the constant term 1.48 is used to achieve consistency of scale parameter at normal distribution [33]. The term $\frac{\left\|\hat{y}_{k}-\hat{y}_{p}\right\|^{2}}{\sigma^{2}}$ is a measure of the discrepancy between full model and submodel. The value of this term is large for the wrong model as compared to the correct model, and it is a good measure to detect the correct models. However, the smallest value of this term indicates that the submodel is closer to the full model, and its value is zero when the submodel itself is the full model. Thus, minimization of $\frac{\left\|\hat{y}_{k}-\hat{y}_{p}\right\|^{2}}{\sigma^{2}}$ is not a proper model selection criterion because this can't accomplish principle of parsimony. Hence, the measure of discrepancy is necessary but not sufficient in model selection.

Most of the model selection criterion expressed as a goodness of fit term plus a measure of complexity. The dimension of the model $(p)$ is a trivial measure of the complexity of the model. Any increasing function of $p$ can be viewed as a complexity measure of a model. However, it ignores the sample size, hence making the resulting criterion inconsistent for instance AIC [16]. Hence, a good complexity measure should be an increasing function of both dimension of the model, $p$ and the sample size, $n$. To make a good criterion, we consider an increasing function of $n$ and $p, C(n, p)$ as a measure of the complexity of the model. The model having smaller value of $A S_{p}$ will be the best model for prediction. Hence, we select the model having smaller $A S_{p}$.

The $A S_{p}$ criterion based on LS estimator and M-estimator with certain $C(n, p)$ are equivalent to Mallows's $C_{p}$ and $S_{p}$ criteria respectively. The $A S_{p}$ criterion can be viewed as a generalization of $C_{p}$ and $S_{p}$ statistic. The $C_{p}, S_{p}$ (or $R C_{p}$ ) criterion selects the model according to 'close to $p$ (or $V_{p}$ )' rule, whereas the $A S_{p}$ criterion selects the model for which its $A S_{p}$ value is minimum. Hence, the model selection using $C_{p}, S_{p}$ and $R C_{p}$ are more intricate as compared to $A S_{p}$.

Proposition 3.1. Least squares estimator based $A S_{p}$ criterion with $C(n, p)=2 p-k$ is equivalent to Mallows's $C_{p}$.

Proof. The $A S_{p}$ criterion based on the LS estimator is defined as

$$
A S_{p}=\frac{\left\|\hat{y}_{k}-\hat{y}_{p}\right\|^{2}}{\sigma^{2}}+C(n, p)
$$

where LS estimator is used to estimate $\hat{y}_{k}$ and $\hat{y}_{p}$ values, and

$$
\begin{aligned}
\left\|\hat{y}_{k}-\hat{y}_{p}\right\|^{2} & =\left\|\left(\hat{y}_{k}-y\right)-\left(\hat{y}_{p}-y\right)\right\|^{2} \\
& =R S S_{k}+R S S_{p}-2\left(\hat{y}_{k}-y\right)^{\prime}\left(\hat{y}_{p}-y\right) .
\end{aligned}
$$

After simplification we get,

$$
\left\|\hat{y}_{k}-\hat{y}_{p}\right\|^{2}=R S S_{k}+R S S_{p}-2 R S S_{k}=R S S_{p}-R S S_{k}[18] .
$$

Hence, $A S_{p}$ criterion with penalty $C(n, p)=2 p-k$ is

$$
A S_{p}=\frac{R S S_{p}-R S S_{k}}{\sigma^{2}}+2 p-k .
$$

Since $\sigma^{2}$ is unknown, it replaced by its suitable estimator $\hat{\sigma}^{2}=\frac{R S S_{k}}{n-k}$. Thus,

$$
\begin{aligned}
A S_{p} & =\frac{R S S_{p}}{\hat{\sigma}^{2}}-(n-2 p) \\
& =C_{p} .
\end{aligned}
$$

Proposition 3.2. M-estimator based $A S_{p}$ criterion with $C(n, p)=2 p-k$ is equivalent to $S_{p}$. 
Proof. The $A S_{p}$ criterion based on the M-estimator with a penalty $C(n, p)=2 p-k$ is given as

$$
\begin{aligned}
A S_{p} & =\frac{\left\|\hat{y}_{k}-\hat{y}_{p}\right\|^{2}}{\sigma^{2}}-(k-2 p) \\
& =S_{p} .
\end{aligned}
$$

Following [16], consider the selected model referred by $M_{\alpha}, \alpha$ denotes the set of selected predictor variables including the intercept. The class of models having all relevant predictor variables is denoted by the class of correct models $\left(\mathcal{M}_{c}\right)$, and the class of wrong models $\left(\mathcal{M}_{w}\right)$ is a class of models in which at least one relevant predictor is missing. A model having only relevant predictors is known as the optimal model, and it is denoted by $M_{\alpha^{o}}$.

Proposition 3.3. For any correct model, $E\left(A S_{p}\right)=\operatorname{tr}\left[\left(H-H_{p}\right)^{\prime}\left(H-H_{p}\right)\right]+C(n, p)$.

Proof. The expected value of $A S_{p}$ is given by,

$$
E\left(A S_{p}\right)=E\left(\frac{1}{\sigma^{2}}\left[y^{\prime}\left(H-H_{p}\right)^{\prime}\left(H-H_{p}\right) y\right]+C(n, p)\right) .
$$

Since, $\left(H-H_{p}\right)^{\prime}\left(H-H_{p}\right)$ is symmetric matrix and $C(n, p)$ is a constant,

$$
\begin{aligned}
E\left(A S_{p}\right) & =\frac{1}{\sigma^{2}} E\left[y^{\prime}\left(H-H_{p}\right)^{\prime}\left(H-H_{p}\right) y\right]+C(n, p) \\
& =\frac{1}{\sigma^{2}}\left\{\sigma^{2} \operatorname{tr}\left[\left(H-H_{p}\right)^{\prime}\left(H-H_{p}\right)\right]+\beta^{\prime} X^{\prime}\left(H-H_{p}\right)^{\prime}\left(H-H_{p}\right) X \beta\right\}+C(n, p) .
\end{aligned}
$$

But, for any correct model, $\beta^{\prime} X^{\prime}\left(H-H_{p}\right)^{\prime}\left(H-H_{p}\right) X \beta=0$. Hence,

$$
E\left(A S_{p}\right)=\operatorname{tr}\left[\left(H-H_{p}\right)^{\prime}\left(H-H_{p}\right)\right]+C(n, p) .
$$

Under following mild regulatory conditions, Theorem 3.6 exhibits consistency property of the proposed $A S_{p}$ criterion for fixed number of predictor variables.

Condition 3.4. For any wrong model $M_{\alpha} \in \mathcal{M}_{w}, \liminf _{n \rightarrow \infty} \frac{\left\|X \beta-X_{\alpha} \beta_{\alpha}\right\|^{2}}{n \sigma^{2}}>0$.

Whenever $M_{\alpha} \in \mathcal{M}_{w}$, it is prospect that the difference $\left\|X \beta-X_{\alpha} \beta_{\alpha}\right\|^{2}$ is large and hence the assumption is justifiable.

Condition 3.5. $C\left(n, p_{\alpha}\right)=o(n)$ and $C\left(n, p_{\alpha}\right) \rightarrow \infty$ as $n \rightarrow \infty, p_{\alpha}$ is a cardinality of a set $\alpha(|\alpha|)$.

The following theorem indicates that, if we choose a model having small $A S_{p}$ value from all possible models, then asymptotically the selected model is an optimal model.

Theorem 3.6 (Consistency Property). Under Conditions 3.4 and 3.5, AS selects the optimal model with probability one, i.e. $\lim _{n \rightarrow \infty} \operatorname{Pr}\left(M_{\alpha}=M_{\alpha^{o}}\right)=1$.

Proof. The proof is divided into two parts. In first part, we show that $A S_{p}$ value of any wrong model is greater than any correct model and in second part, $A S_{p}$ value of optimal model $M_{\alpha^{o}}$ is smaller among the class of correct models $\mathcal{M}_{c}$. For any model $M_{\alpha}, \hat{y}_{p_{\alpha}}$ is a 
vector of predicted values of $y$ and hence

$$
\begin{aligned}
\left\|\hat{y}_{k}-\hat{y}_{p_{\alpha}}\right\|^{2}= & \left\|X \hat{\beta}-X_{\alpha} \hat{\beta}_{\alpha}\right\|^{2} \\
= & \|X \hat{\beta}-X \beta\|^{2}+\left\|X_{\alpha} \hat{\beta}_{\alpha}-X \beta\right\|^{2}-2(X \hat{\beta}-X \beta)^{\prime}\left(X_{\alpha} \hat{\beta}_{\alpha}-X \beta\right) \\
= & \|X \hat{\beta}-X \beta\|^{2}+\left\|X_{\alpha} \hat{\beta}_{\alpha}-X_{\alpha} \beta_{\alpha}\right\|^{2}+\left\|X \beta-X_{\alpha} \beta_{\alpha}\right\|^{2} \\
& -2\left(X_{\alpha} \hat{\beta}_{\alpha}-X_{\alpha} \beta_{\alpha}\right)^{\prime}\left(X \beta-X_{\alpha} \beta_{\alpha}\right)-2(X \hat{\beta}-X \beta)^{\prime}\left(X_{\alpha} \hat{\beta}_{\alpha}-X_{\alpha} \beta_{\alpha}\right) \\
& +2(X \hat{\beta}-X \beta)^{\prime}\left(X \beta-X_{\alpha} \beta_{\alpha}\right) \\
= & \|X \hat{\beta}-X \beta\|^{2}+\left\|X_{\alpha} \hat{\beta}_{\alpha}-X_{\alpha} \beta_{\alpha}\right\|^{2}+\left\|X \beta-X_{\alpha} \beta_{\alpha}\right\|^{2}+\zeta_{1}+\zeta_{2}+\zeta_{3} .
\end{aligned}
$$

Since, $\hat{\beta}_{\alpha}-\beta_{\alpha}=o_{p}(1)$ [24] and by law of large numbers, $\zeta_{1}=o_{p}(n), \zeta_{2}=o_{p}(n)$ and $\zeta_{3}=o_{p}(n)$. Thus,

$$
\left\|\hat{y}_{k}-\hat{y}_{p_{\alpha}}\right\|^{2}=\|X \hat{\beta}-X \beta\|^{2}+\left\|X_{\alpha} \hat{\beta}_{\alpha}-X_{\alpha} \beta_{\alpha}\right\|^{2}+\left\|X \beta-X_{\alpha} \beta_{\alpha}\right\|^{2}+o_{p}(n) .
$$

Similarly, under some regulatory conditions, we have $\left\|X_{\alpha} \hat{\beta}_{\alpha}-X_{\alpha} \beta_{\alpha}\right\|^{2}=O_{p}(1)[9,20,24]$. For any $M_{\alpha} \in \mathcal{M}_{c}, X \beta=X_{\alpha} \beta_{\alpha}$ and by Cauchy-Schwartz inequality

$$
\begin{aligned}
\left\|\hat{y}_{k}-\hat{y}_{p_{\alpha}}\right\|^{2} & =\|X \hat{\beta}-X \beta\|^{2}+\left\|X_{\alpha} \hat{\beta}_{\alpha}-X_{\alpha} \beta_{\alpha}\right\|^{2}-2(X \hat{\beta}-X \beta)^{\prime}\left(X_{\alpha} \hat{\beta}_{\alpha}-X_{\alpha} \beta_{\alpha}\right) \\
& \leq\|X \hat{\beta}-X \beta\|^{2}+\left\|X_{\alpha} \hat{\beta}_{\alpha}-X_{\alpha} \beta_{\alpha}\right\|^{2}+2\|X \hat{\beta}-X \beta\|^{2}\left\|X_{\alpha} \hat{\beta}_{\alpha}-X_{\alpha} \beta_{\alpha}\right\|^{2} \\
& =O_{p}(1) .
\end{aligned}
$$

Let $A S_{p_{\alpha}}$ and $A S_{p_{\alpha^{*}}}$ are values of the $A S_{p}$ of any wrong model $M_{\alpha} \in \mathcal{M}_{w}$ and correct model $M_{\alpha^{*}} \in \mathcal{M}_{c}$ respectively. By Condition 3.4, 3.5 and combining (3.3), (3.4),

$$
\begin{aligned}
\liminf _{n \rightarrow \infty} \operatorname{Pr}\left(A S_{p_{\alpha}}>A S_{p_{\alpha^{*}}}\right) & =\liminf _{n \rightarrow \infty} \operatorname{Pr}\left(\frac{\left\|X \beta-X_{\alpha} \beta_{\alpha}\right\|^{2}}{\sigma^{2}}+o_{p}(n)>0\right) \\
& \geq \operatorname{Pr}\left(\liminf _{n \rightarrow \infty} \frac{\left\|X \beta-X_{\alpha} \beta_{\alpha}\right\|^{2}}{\sigma^{2}}+o_{p}(n)>0\right) \\
& =1 .
\end{aligned}
$$

Thus, the value of $A S_{p}$ of a wrong model is greater than any correct model belongs to the class of correct models for large sample size. In the light of Equation (3.5), it is sufficient to show that the $A S_{p}$ value of the optimal model is small among the class of correct models $\mathcal{M}_{c}$ to complete the proof of consistency property. By Condition 3.5, $C\left(n, p_{\alpha}\right) \rightarrow \infty$ as $n \rightarrow \infty$ and $C\left(n, p_{\alpha^{*}}\right)-C\left(n, p_{\alpha^{o}}\right) \geq 0$ because $p_{\alpha^{*}} \geq p_{\alpha^{o}}$ for any correct model. From Equation (3.4), we have

$$
\begin{aligned}
A S_{p_{\alpha^{*}}}-A S_{p_{\alpha^{o}}} & =\frac{\left\|\hat{y}_{k}-\hat{y}_{p_{\alpha^{*}}}\right\|^{2}}{\sigma^{2}}-\frac{\left\|\hat{y}_{k}-\hat{y}_{p_{\alpha^{o}}}\right\|^{2}}{\sigma^{2}}+C\left(n, p_{\alpha^{*}}\right)-C\left(n, p_{\alpha^{o}}\right) \\
& =O_{p}(1)+C\left(n, p_{\alpha^{*}}\right)-C\left(n, p_{\alpha^{o}}\right) \\
& \geq 0 .
\end{aligned}
$$

This proves that, the $A S_{p}$ is a consistent criterion.

\section{Performance of $A S_{p}$ criterion}

In this section, we evaluate the performance of $A S_{p}$ through a simulation study by considering three regression models and six penalty functions (Table 1). We have generated data and introduced vertical outliers and leverage points in this data using the procedure given in the Section 2. The good leverage point does not affect estimator of regression 
parameters and the $A S_{p}$ criterion based on this estimator. Therefore, throughout the entire simulation study, bad leverage points are considered and are mentioned as leverage points. The different combinations of the number of vertical outliers and the number of leverage points have considered in this simulation study. The Huber $\psi(\cdot)$ function is widely used for robust parameter estimation in linear regression, and the tunning constant 1.345 achieves high efficiency over LS estimator in normal case [20]. The $A S_{p}$ statistic for all possible submodels calculated using Huber's $\psi(\cdot)$ function with tuning constant 1.345.

Table 1. Models and Penalty functions.

\begin{tabular}{cll}
\hline Sr. No. & \multicolumn{1}{c}{ Model } & Penalty function $C(n, p)$ \\
\hline 1. & $M_{1}: y=5+2 X_{1}+3 X_{2}+0 X_{3}+\varepsilon$ & $\mathrm{P} 1=3 p$ \\
2. & $M_{2}: y=4+3 X_{1}-2 X_{2}+7 X_{3}+0 X_{4}+0 X_{5}+\varepsilon$ & $\mathrm{P} 2=2 p \log (p)$ \\
3. & $M_{3}: y=3+2.5 X_{1}+1.7 X_{2}-6 X_{3}+8 X_{4}+0 X_{5}+0 X_{6}+0 X_{7}+\varepsilon$ & $\mathrm{P} 3=p \log (n)$ \\
& & $\mathrm{P} 4=p(\log (n)+1)$ \\
& & $\mathrm{P} 5=6 p \log (\log (n))$ \\
& & $\mathrm{P} 6=p \sqrt{n}$ \\
\hline
\end{tabular}

\section{1. $A S_{p}$ criterion with different penalties}

In Table 2 and 3, the percentage of optimal model selection based on 1000 runs for different combinations of penalties, sample sizes, and models has recorded. For model $M_{1}$, all penalties work well in case of clean data (0-Vertical outliers and 0-Leverage points) and select an optimal model with at least $75 \%$ when the sample size is 50 . After adding vertical outliers/and leverage points in the data still, $A S_{p}$ work well and selects an optimal model with up to $81.4 \%$ in the presence of vertical outlier only, $65.6 \%$ in the presence of leverage point only, $62.7 \%$ in the presence of both vertical outlier and leverage point for sample size 50. Moreover, the $A S_{p}$ works satisfactorily for other combinations of the number of vertical outliers and leverage points. These percentages of optimal model selection increase with increasing sample size. The similar results have obtained from model $M_{2}$.

For model $M_{3}$, the $A S_{p}$ criterion selects the optimal model with at least $59 \%$ for clean data and sample size 50; but as sample size increases this percentage increases. $A S_{p}$ performs better in the presence of vertical outliers/and leverage points, and select optimal model with more than $90 \%$ for large sample size. It is observed that, the performance of the $A S_{p}$ criterion is based on ratio $k / n$. The percentage of optimal model selection increases as $k / n$ decreases.

Hence, for small sample size and in the presence of vertical outliers/and leverage points, $A S_{p}$ performs satisfactorily. As the sample size increases, the optimal model selection percentage increases to $100 \%$ for P3, P4, P5 and P6 penalties. Thus, the simulation results show that the proposed criterion is consistent. Also, the $A S_{p}$ criterion with $\mathrm{P} 1$ and $\mathrm{P} 2$ penalties select the optimal model preciously in the presence of vertical outliers/and leverage points for large sample size. Thus, the simulation study indicates that the proposed criterion performs well and selects the optimal model more preciously for a large sample size.

\subsection{Comparative study of $A S_{p}$ with $C_{p}, S_{p}$ and $R C_{p}$}

The comparative study of $A S_{p}$ and other criteria $\left(C_{p}, S_{p}\right.$ and $\left.R C_{p}\right)$ in the presence of vertical outlier and leverage point is carried out through model selection ability for same simulation design and $M_{1}, M_{2}$ models. The model selection ability of these criteria has presented in Figure 3 by using 100 simulated datasets. In Figure 3, P1, P2, P3, P4, P5 and $\mathrm{P} 6$ refers to $A S_{p}$ criterion with $\mathrm{P} 1, \mathrm{P} 2, \mathrm{P} 3, \mathrm{P} 4, \mathrm{P} 5$ and $\mathrm{P} 6$ penalty function respectively. This simulation study shows that the simultaneous occurrence of a vertical outlier and a 
leverage point effect on the performance of $C_{p}, S_{p}, R C_{p}$ criteria, and most of the times $C_{p}$ selects wrong or overfitted model. Mostly $S_{p}$ select overfitted model, but the number of the wrong model selected by $S_{p}$ is small as compared to $C_{p}$. Another one robust criterion $R C_{p}$ select optimal model more preciously as compare to $C_{p}$ and $S_{p}$, but it performs poorly as compared to $A S_{p}$. The difference between overfitted models selected by $R C_{p}$ and $A S_{p}$ is notable. Thus, the $A S_{p}$ criterion increases the optimal model selection percentage by at least $50 \%$ relative to others. As the sample size increases, the probability of selecting the wrong model using the above criteria approaches to zero. However, the $A S_{p}$ selects an optimal model with a large percentage as compared to $C_{p}, S_{p}$ and $R C_{p}$. In conclusion, the $A S_{p}$ has more model selection ability as compared to others.

Table 2. Percentage of selecting optimal model (1000 runs).

\begin{tabular}{|c|c|c|c|c|c|c|c|c|c|c|c|c|c|}
\hline \multirow{2}{*}{ Model } & \multirow{2}{*}{$\mathrm{n}$} & \multicolumn{2}{|c|}{ 0-Vertical } & \multirow{2}{*}{$\frac{\text { outliers }}{\text { P3 }}$} & \multirow{2}{*}{$\begin{array}{c}\text { and } \\
\mathrm{P} 4\end{array}$} & \multirow{2}{*}{\multicolumn{2}{|c|}{$\begin{array}{cc}\text { 0-Leverage points } \\
\text { P5 } & \text { P6 }\end{array}$}} & \multicolumn{2}{|c|}{ 1-Vertical } & \multirow{2}{*}{$\frac{\text { outlier }}{\text { P3 }}$} & and 0 & )-Leverage & \multirow{2}{*}{$\frac{\text { points }}{\mathrm{P} 6}$} \\
\hline & & $\mathrm{P} 1$ & $\mathrm{P} 2$ & & & & & $\mathrm{P} 1$ & P2 & & $\mathrm{P} 4$ & $\mathrm{P} 5$ & \\
\hline \multirow{5}{*}{$M_{1}$} & 50 & 75.4 & 85.4 & 81.8 & 85.6 & 84.7 & 86.1 & 68.5 & 79.9 & 75.6 & 80.2 & 79.9 & 81.4 \\
\hline & 70 & 77.7 & 87.8 & 86.7 & 89.8 & 91.9 & 92.2 & 74.6 & 86.8 & 85.3 & 89.1 & 91.7 & 91.8 \\
\hline & 100 & 78.2 & 89.4 & 89.8 & 93.0 & 97.1 & 97.4 & 74.0 & 86.8 & 87.2 & 90.7 & 97.0 & 97.2 \\
\hline & 200 & 81.1 & 89.4 & 92.9 & 96.0 & 99.3 & 99.8 & 79.0 & 90.3 & 93.5 & 95.9 & 99.5 & 99.8 \\
\hline & 300 & 79.3 & 91.1 & 94.6 & 97.2 & 99.6 & 100.0 & 79.6 & 90.5 & 95.0 & 96.5 & 99.4 & 99.8 \\
\hline \multirow{5}{*}{$M_{2}$} & 50 & 75.0 & 86.1 & 81.6 & 85.2 & 85.2 & 86.0 & 65.5 & 80.4 & 74.1 & 79.5 & 81.2 & 82.5 \\
\hline & 70 & 78.1 & 89.4 & 87.1 & 89.7 & 93.3 & 93.3 & 75.1 & 87.3 & 84.0 & 88.2 & 92.0 & 92.3 \\
\hline & 100 & 77.7 & 91.2 & 89.7 & 93.3 & 97.7 & 98.4 & 73.0 & 89.3 & 87.3 & 91.9 & 96.8 & 97.5 \\
\hline & 200 & 81.3 & 92.2 & 92.8 & 95.6 & 99.1 & 99.9 & 79.7 & 92.1 & 93.0 & 95.4 & 98.8 & 99.8 \\
\hline & 300 & 80.0 & 91.9 & 94.0 & 96.6 & 99.4 & 100.0 & 79.4 & 91.5 & 94.3 & 96.2 & 99.4 & 99.8 \\
\hline \multirow{5}{*}{$M_{3}$} & 50 & 59.1 & 72.5 & 66.7 & 70.4 & 67.4 & 69.7 & 55.0 & 70.3 & 64.0 & 68.0 & 65.0 & 67.2 \\
\hline & 70 & 66.1 & 84.0 & 77.8 & 83.0 & 82.3 & 82.8 & 61.0 & 80.0 & 73.1 & 79.1 & 81.1 & 81.5 \\
\hline & 100 & 70.0 & 88.6 & 85.1 & 89.3 & 93.8 & 92.3 & 66.6 & 86.2 & 81.7 & 86.9 & 93.8 & 92.6 \\
\hline & 200 & 73.3 & 91.7 & 91.0 & 95.0 & 99.0 & 99.8 & 70.7 & 90.1 & 89.3 & 93.9 & 98.6 & 99.7 \\
\hline & 300 & 72.3 & 91.7 & 92.7 & 95.7 & 99.3 & 99.8 & 74.5 & 92.0 & 92.9 & 95.5 & 99.4 & 99.8 \\
\hline \multirow{2}{*}{ Model } & \multirow{2}{*}{$\mathrm{n}$} & \multicolumn{2}{|c|}{ 0-Vertical } & outliers & and & 1-Lev & ge point & \multicolumn{2}{|c|}{ 1-Vertical } & outlier & and & 1-Leve & e point \\
\hline & & $\mathrm{P} 1$ & $\mathrm{P} 2$ & P3 & $\mathrm{P} 4$ & $\mathrm{P} 5$ & P6 & $\mathrm{P} 1$ & P2 & P3 & $\mathrm{P} 4$ & P5 & P6 \\
\hline \multirow{5}{*}{$M_{1}$} & 50 & 54.6 & 64.1 & 60.9 & 63.9 & 64.7 & 65.6 & 49.0 & 58.3 & 54.8 & 58.6 & 62.7 & 61.6 \\
\hline & 70 & 57.9 & 69.8 & 68.1 & 73.4 & 79.8 & 79.3 & 55.4 & 69.6 & 68.2 & 72.2 & 79.7 & 79.8 \\
\hline & 100 & 63.3 & 76.5 & 76.9 & 81.9 & 90.7 & 91.2 & 58.8 & 71.5 & 72.4 & 78.3 & 87.9 & 88.9 \\
\hline & 200 & 71.8 & 82.4 & 86.4 & 90.7 & 96.8 & 99.1 & 69.0 & 80.4 & 85.7 & 89.9 & 96.2 & 98.6 \\
\hline & 300 & 73.4 & 84.7 & 89.3 & 93.1 & 98.6 & 99.9 & 74.5 & 85.6 & 91.8 & 94.9 & 98.1 & 99.7 \\
\hline \multirow{5}{*}{$M_{2}$} & 50 & 52.7 & 65.7 & 59.7 & 65.1 & 71.9 & 70.6 & 45.8 & 60.8 & 53.0 & 59.6 & 68.1 & 64.9 \\
\hline & 70 & 56.1 & 71.0 & 66.2 & 72.4 & 82.1 & 81.4 & 55.7 & 69.3 & 66.0 & 71.2 & 82.4 & 82.0 \\
\hline & 100 & 60.1 & 77.7 & 75.0 & 80.9 & 90.9 & 91.8 & 59.8 & 77.4 & 74.7 & 80.4 & 90.5 & 91.4 \\
\hline & 200 & 68.6 & 85.1 & 86.4 & 90.8 & 96.9 & 99.0 & 69.8 & 85.0 & 86.8 & 90.2 & 96.8 & 99.1 \\
\hline & 300 & 73.0 & 88.9 & 91.7 & 94.5 & 98.6 & 99.8 & 74.3 & 86.4 & 90.1 & 92.4 & 97.7 & 99.9 \\
\hline \multirow{5}{*}{$M_{3}$} & 50 & 34.8 & 49.0 & 41.8 & 46.5 & 50.4 & 50.6 & 33.7 & 47.1 & 41.0 & 44.9 & 45.7 & 47.0 \\
\hline & 70 & 49.7 & 67.0 & 60.1 & 65.5 & 69.9 & 69.4 & 42.1 & 62.0 & 53.3 & 60.6 & 65.2 & 64.9 \\
\hline & 100 & 49.6 & 71.2 & 65.7 & 71.6 & 82.4 & 82.7 & 47.2 & 69.4 & 63.9 & 70.1 & 80.4 & 81.6 \\
\hline & 200 & 61.2 & 83.7 & 82.6 & 88.2 & 95.2 & 98.1 & 60.2 & 83.0 & 82.3 & 87.6 & 95.6 & 98.2 \\
\hline & 300 & 64.3 & 84.3 & 85.5 & 89.7 & 97.1 & 99.8 & 63.3 & 86.5 & 87.6 & 90.4 & 96.7 & 99.5 \\
\hline
\end{tabular}


Table 3. Percentage of selecting optimal model (1000 runs).

\begin{tabular}{|c|c|c|c|c|c|c|c|c|c|c|c|c|c|}
\hline \multirow{2}{*}{ Model } & \multirow{2}{*}{$\mathrm{n}$} & \multicolumn{2}{|c|}{ 0-Vertical } & \multirow{2}{*}{$\frac{\text { outliers }}{\text { P3 }}$} & \multirow{2}{*}{$\frac{\text { and }}{\mathrm{P} 4}$} & \multirow{2}{*}{\multicolumn{2}{|c|}{$\begin{array}{cc}\text { 2-Leverage points } \\
\text { P5 } & \text { P6 }\end{array}$}} & \multicolumn{2}{|c|}{ 2-Vertical } & \multirow{2}{*}{$\frac{\text { outliers }}{\text { P3 }}$} & \multirow{2}{*}{$\frac{\text { and }}{\mathrm{P} 4}$} & \multirow{2}{*}{$\frac{0 \text {-Leverage }}{\text { P5 }}$} & \multirow{2}{*}{$\frac{\mathrm{e} \text { points }}{\mathrm{P} 6}$} \\
\hline & & P1 & P2 & & & & & $\mathrm{P} 1$ & $\mathrm{P} 2$ & & & & \\
\hline \multirow{5}{*}{$M_{1}$} & 50 & 52.3 & 58.0 & 55.2 & 55.6 & 51.8 & 53.7 & 82.8 & 87.3 & 85.0 & 86.7 & 79.3 & 82.7 \\
\hline & 70 & 62.7 & 71.0 & 69.9 & 72.2 & 72.5 & 72.3 & 85.7 & 91.5 & 90.5 & 91.6 & 89.7 & 90.6 \\
\hline & 100 & 69.0 & 77.7 & 77.9 & 81.2 & 86.5 & 86.0 & 88.3 & 94.3 & 94.3 & 96.3 & 97.5 & 96.8 \\
\hline & 200 & 73.6 & 81.1 & 84.4 & 87.6 & 94.8 & 97.6 & 88.5 & 93.5 & 95.1 & 96.8 & 99.0 & 99.9 \\
\hline & 300 & 81.5 & 89.2 & 92.9 & 94.4 & 98.2 & 99.7 & 90.2 & 94.9 & 96.9 & 98.6 & 99.9 & 100.0 \\
\hline \multirow{5}{*}{$M_{2}$} & 50 & 44.0 & 54.5 & 50.4 & 53.3 & 59.6 & 59.6 & 69.2 & 79.4 & 75.5 & 78.3 & 76.0 & 78.4 \\
\hline & 70 & 46.7 & 60.2 & 55.6 & 61.1 & 71.7 & 70.9 & 75.4 & 88.1 & 84.7 & 87.4 & 88.3 & 88.7 \\
\hline & 100 & 51.6 & 67.5 & 64.7 & 71.0 & 82.6 & 84.6 & 78.8 & 91.7 & 89.4 & 94.0 & 97.4 & 97.3 \\
\hline & 200 & 63.4 & 79.2 & 81.0 & 85.7 & 93.1 & 96.3 & 79.0 & 91.9 & 92.9 & 95.8 & 99.6 & 100.0 \\
\hline & 300 & 66.1 & 82.7 & 86.7 & 90.3 & 96.8 & 98.9 & 81.1 & 93.6 & 95.5 & 97.6 & 99.5 & 100.0 \\
\hline \multirow{5}{*}{$M_{3}$} & 50 & 30.4 & 36.7 & 33.5 & 35.6 & 33.9 & 34.7 & 62.0 & 66.9 & 66.0 & 65.9 & 54.3 & 58.9 \\
\hline & 70 & 33.6 & 46.9 & 42.8 & 46.0 & 48.9 & 49.4 & 66.3 & 81.0 & 75.3 & 80.3 & 76.1 & 77.0 \\
\hline & 100 & 42.5 & 62.2 & 57.7 & 62.5 & 71.1 & 71.3 & 73.3 & 90.6 & 87.2 & 90.3 & 92.3 & 90.4 \\
\hline & 200 & 51.1 & 73.5 & 72.8 & 77.8 & 90.5 & 95.1 & 71.9 & 91.8 & 91.1 & 94.5 & 98.9 & 99.4 \\
\hline & 300 & 54.0 & 76.9 & 78.7 & 83.0 & 94.9 & 98.7 & 74.3 & 92.3 & 92.9 & 95.6 & 99.3 & 100.0 \\
\hline \multirow{2}{*}{ Model } & \multirow{2}{*}{$\mathrm{n}$} & \multicolumn{2}{|c|}{ 1-Vertical } & outlier & and 2 & -Lever & age points & $2-\mathrm{Ve}$ & tical & outliers & and & 1-Leve & rage point \\
\hline & & $\mathrm{P} 1$ & P2 & P3 & $\mathrm{P} 4$ & $\mathrm{P} 5$ & $\mathrm{P} 6$ & $\mathrm{P} 1$ & $\mathrm{P} 2$ & P3 & $\mathrm{P} 4$ & $\mathrm{P} 5$ & P6 \\
\hline & 50 & 52.1 & 58.3 & 55.5 & 57.0 & 53.2 & 55.3 & 65.9 & 71.8 & 69.7 & 70.7 & 68.8 & 70.5 \\
\hline & 70 & 60.5 & 68.0 & 66.4 & 67.4 & 68.2 & 68.4 & 77.0 & 84.0 & 82.0 & 84.5 & 84.5 & 85.0 \\
\hline$M_{1}$ & 100 & 65.7 & 75.9 & 76.1 & 80.0 & 85.5 & 86.0 & 78.5 & 85.4 & 85.4 & 88.7 & 93.4 & 94.0 \\
\hline & 200 & 76.0 & 84.4 & 87.4 & 89.8 & 95.3 & 97.9 & 84.1 & 91.2 & 93.1 & 95.5 & 98.4 & 99.5 \\
\hline & 300 & 79.7 & 87.6 & 91.4 & 93.6 & 97.4 & 99.2 & 84.2 & 91.4 & 95.1 & 96.8 & 98.8 & 99.8 \\
\hline & 50 & 39.8 & 54.5 & 48.0 & 53.5 & 61.9 & 59.7 & 54.5 & 66.9 & 61.0 & 65.9 & 67.8 & 68.0 \\
\hline & 70 & 44.7 & 59.6 & 54.7 & 60.9 & 70.3 & 70.0 & 60.1 & 73.3 & 69.1 & 74.2 & 81.5 & 81.2 \\
\hline$M_{2}$ & 100 & 51.6 & 69.1 & 65.8 & 72.2 & 82.5 & 84.4 & 64.2 & 81.0 & 78.7 & 84.0 & 91.8 & 93.2 \\
\hline & 200 & 61.9 & 78.5 & 80.4 & 84.3 & 93.8 & 96.5 & 72.0 & 86.5 & 87.5 & 90.6 & 97.2 & 99.3 \\
\hline & 300 & 68.2 & 82.5 & 85.1 & 88.1 & 96.5 & 99.6 & 75.4 & 89.5 & 92.2 & 94.6 & 98.9 & 99.9 \\
\hline & 50 & 26.6 & 36.1 & 31.8 & 35.1 & 34.0 & 35.3 & 36.4 & 47.6 & 42.4 & 45.5 & 43.7 & 44.6 \\
\hline & 70 & 35.3 & 48.2 & 43.3 & 47.5 & 49.6 & 50.3 & 47.7 & 62.8 & 57.2 & 61.8 & 62.7 & 63.0 \\
\hline$M_{3}$ & 100 & 40.3 & 58.7 & 53.9 & 59.3 & 68.1 & 69.2 & 56.0 & 74.8 & 69.9 & 75.5 & 82.7 & 82.1 \\
\hline & 200 & 50.4 & 73.6 & 73.0 & 78.8 & 90.5 & 94.4 & 62.6 & 84.9 & 84.3 & 89.5 & 96.6 & 98.1 \\
\hline & 300 & 53.1 & 77.9 & 79.8 & 84.7 & 94.7 & 99.0 & 64.8 & 85.3 & 86.9 & 90.2 & 97.4 & 99.5 \\
\hline Model & $\mathrm{n}$ & & & & & 2-Verti & al outliers a & d 2-L & verage & e points & & & \\
\hline Moder & $\mathrm{n}$ & & & $\mathrm{P}$ & & & P3 & & & $\mathrm{P5}$ & & & $\mathrm{P} 6$ \\
\hline & 50 & 45 & & 51 & & & 48.6 & & & 48. & & & 49.3 \\
\hline & 70 & 61 & & 67 & & & 64.8 & & & 68. & & & 68.5 \\
\hline$M_{1}$ & 100 & 66 & & 75 & & & 75.6 & & & 83. & & & 83.4 \\
\hline & 200 & 77 & & 83 & & & 86.8 & & & 95. & & & 98.1 \\
\hline & 300 & & & 88 & & & 92.3 & & & 97. & & & 99.7 \\
\hline & 50 & 38 & & 52 & & & 44.9 & & & 58. & & & 58.1 \\
\hline & 70 & 44 & & 58 & & & 53.5 & & & 69. & & & 69.5 \\
\hline$M_{2}$ & 100 & 49 & & 64 & & & 62.2 & & & 81. & & & 81.8 \\
\hline & 200 & 59 & & 74 & & & 76.2 & & & 90. & & & 95.2 \\
\hline & 300 & 63 & & 79 & & & 84.3 & & & 95. & & & 99.0 \\
\hline & 50 & & & 32 & & & 28.2 & & & 33. & & & 33.4 \\
\hline & 70 & 33 & & 48 & & & 42.3 & & & 50. & & & 50.6 \\
\hline$M_{3}$ & 100 & 39 & & 56 & .3 & & 51.9 & & & 67. & & & 68.3 \\
\hline & 200 & 50 & & 72 & & & 71.8 & & & 90. & & & 95.2 \\
\hline & 300 & 55 & & 79 & & & 81.2 & & & 95. & & & 99.1 \\
\hline
\end{tabular}


(a) $n=50$, Model $=M_{1}$

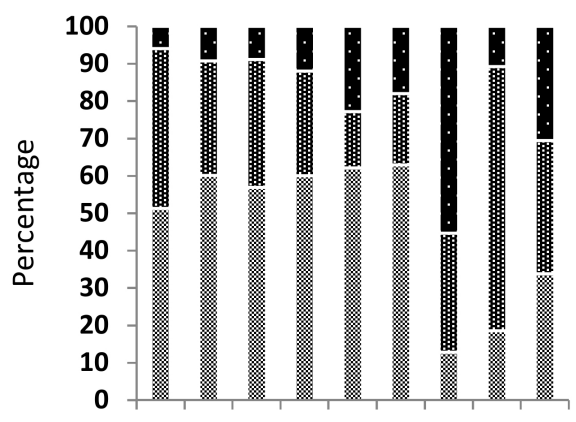

P1 P2 P3 P4 P5 P6 Cp Sp RCp

Criteria

(c) $n=70$, Model $=M_{1}$

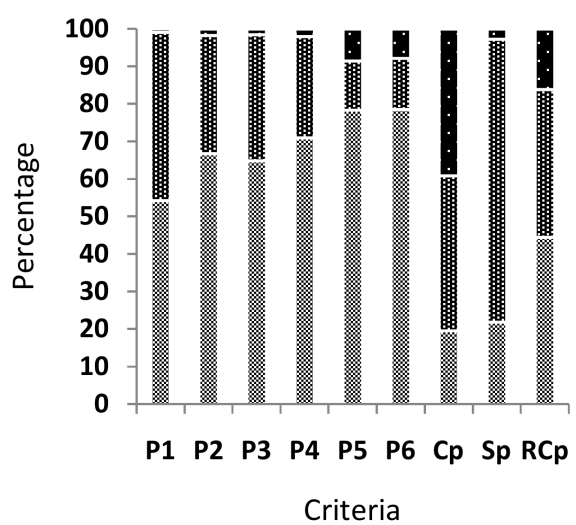

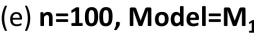

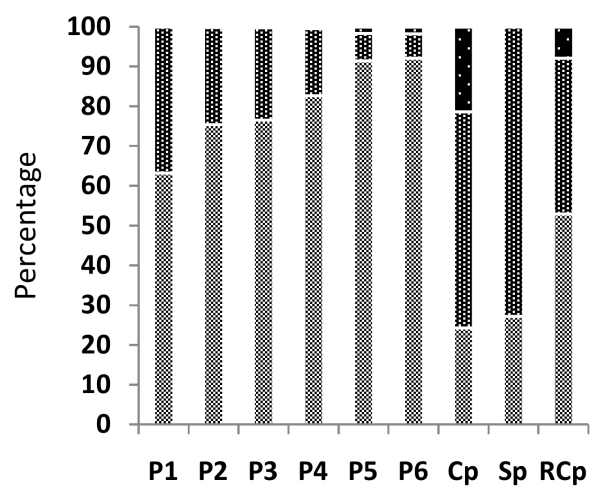

Criteria (b) $n=50$, Model $=M_{2}$

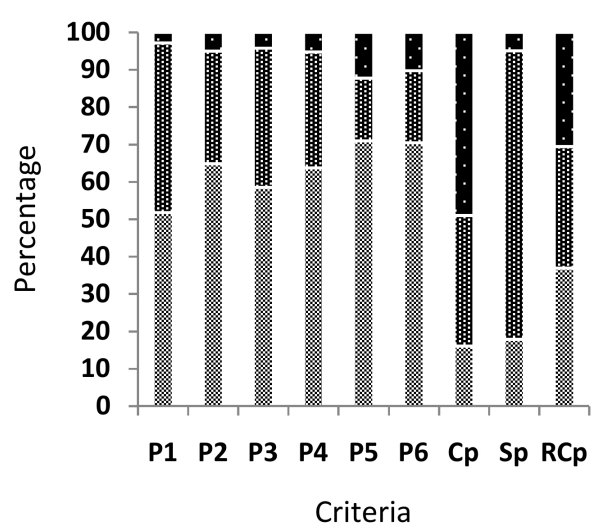

(d) $\mathbf{n = 7 0}$, Model=M

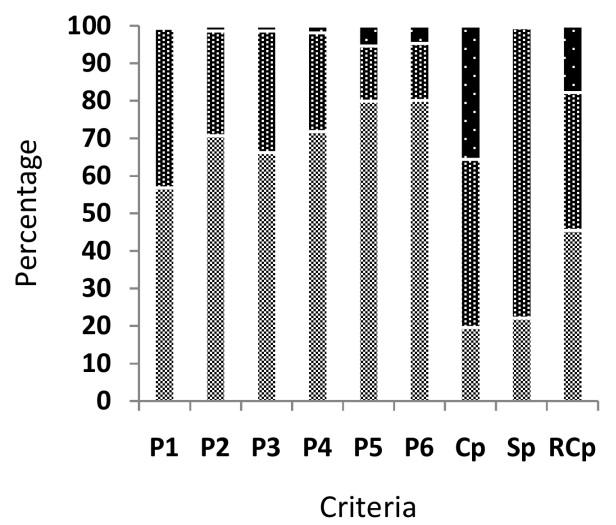

(f) $n=100$, Model $=M_{2}$

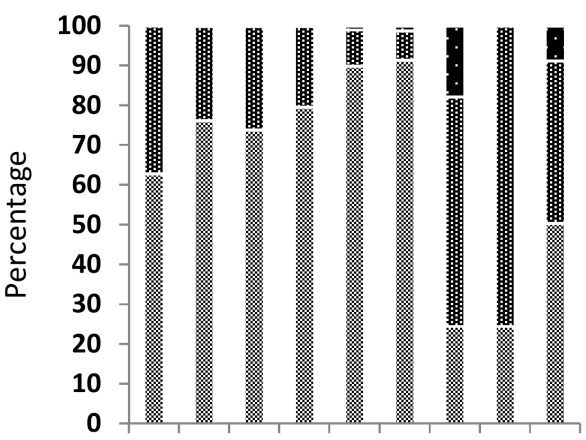

$\begin{array}{lllllllll}\text { P1 } & \text { P2 } & \text { P3 } & \text { P4 } & \text { P5 } & \text { P6 } & \text { Cp } & \text { Sp } & \text { RCp }\end{array}$

Criteria

Continue.. 
(g) $n=200$, Model $=M_{1}$

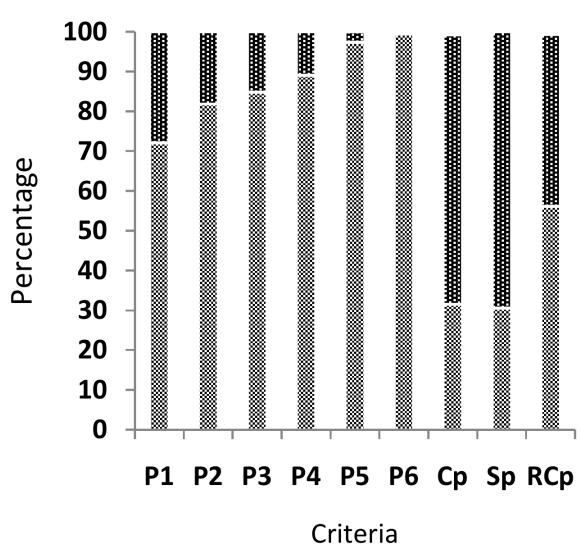

(i) $n=300$, Model $=M_{1}$

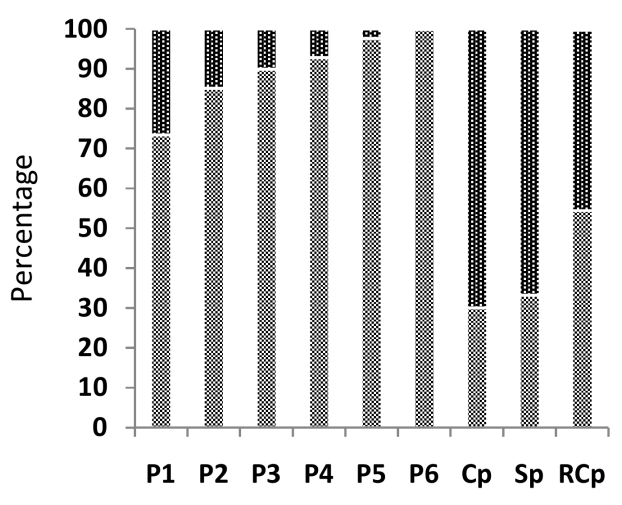

Criteria (h) $\mathbf{n}=\mathbf{2 0 0}$, Model $=\mathbf{M}_{\mathbf{2}}$

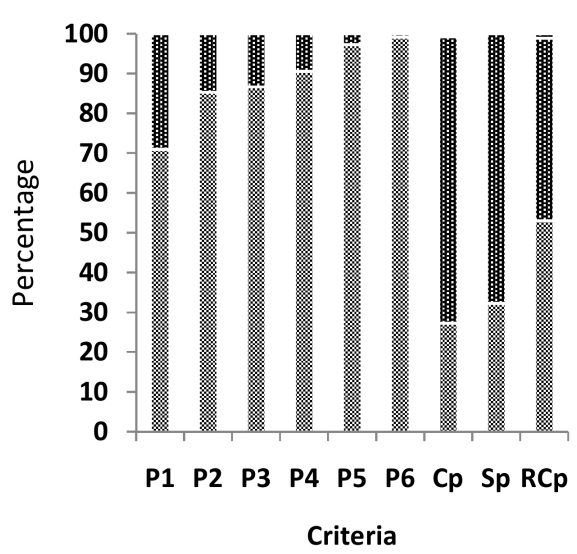

(j) $n=300$, Model $=M_{2}$

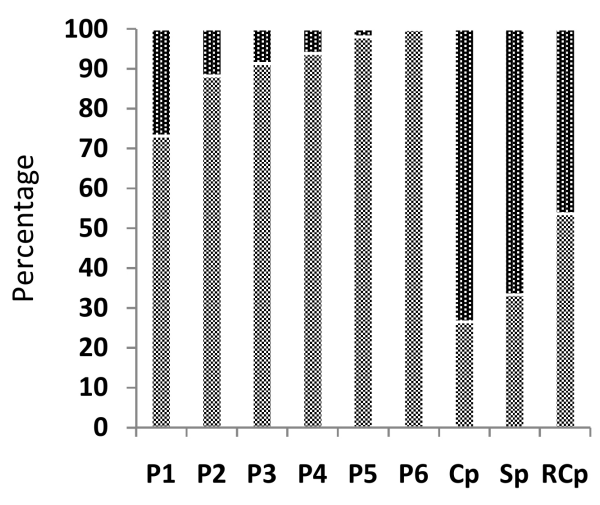

Criteria

Optimal Model, 堽 Overfitted Model, ㅁ Wrong Model

Figure 3. (a)-(j) Model selection ability of $A S_{p}, C_{p}, S_{p}$ and $R C_{p}$.

\subsection{Example: Agglomeration in Bayer precipitation data}

Sommer and Staudte [31] and Bab-Hadiashar and Suter [5] analyzed Bayer precipitation data on the agglomeration of aluminium trihydroxide $\left(\mathrm{Al}(\mathrm{OH})_{3}\right)$ crystals by means of the Bayer precipitation process. Data has 51 observations on the response variable $y$ (An agglomeration of crystals with size exceeding 45 microns, i.e. the difference between the percentage of crystals exceeding this size, which leave the agglomerator tank and those which enter it) and nine predictor variables $X_{1}-X_{9}[5,31]$. Figure 4 shows that, the data has 11 vertical outliers, 6 bad leverage points and 5 good leverage points.

For this data, we compared the performance of non-robust as well as robust criteria. The $C_{p}$ and $R C_{p}$ recommend several submodels with three or more variables, and $S_{p}$ suggests model containing at least five variables (Figure 5). Whereas, the $A S_{p}$ would consider five predictor variables $X_{1}, X_{3}, X_{4}, X_{5}, X_{8}$. Thus, the model having $X_{1}, X_{3}, X_{4}, X_{5}, X_{8}$ variables seems to be "good", according to $A S_{p}$ criterion with different penalties (Table 4). Sommer and Staudte [31] suggest $X_{1}, X_{3}, X_{4}, X_{5}, X_{7}, X_{8}$ predictor variables using $R C_{p}$ with Mallows weight, and Bab-Hadiashar and Suter [5] suggest three best subsets $\left(X_{1}, X_{3}, X_{4}, X_{5}, X_{8}\right),\left(X_{1}, X_{3}, X_{4}, X_{5}, X_{7}, X_{8}\right)$ and $\left(X_{1}, X_{3}, X_{4}, X_{5}, X_{7}, X_{8}, X_{9}\right)$ for the same data. 


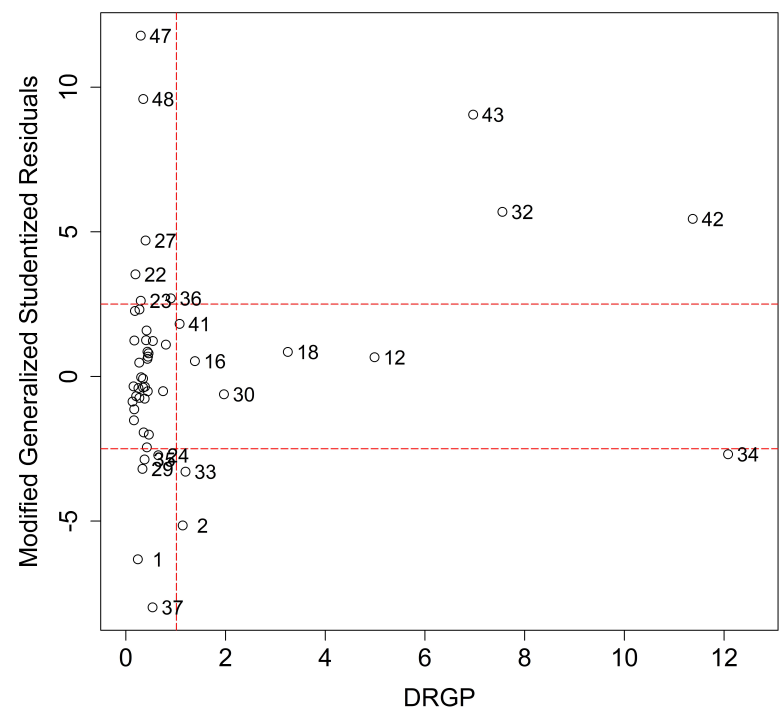

Figure 4. MGt-DRGP plot for Bayer Precipitation data.

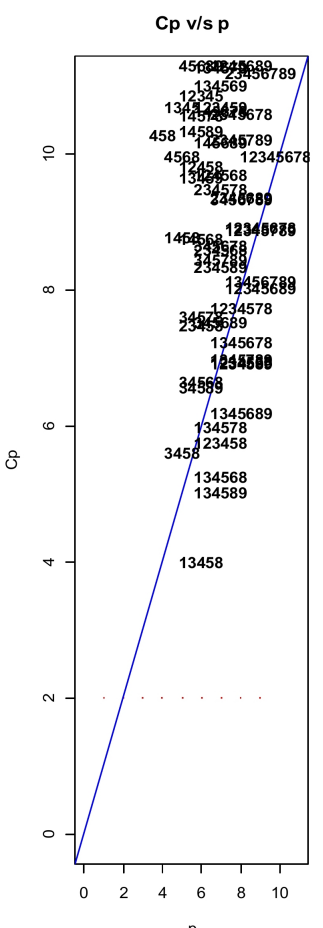

(a)

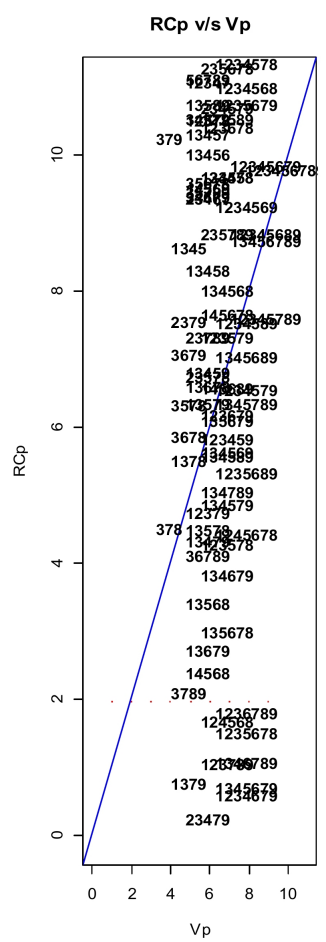

(b)

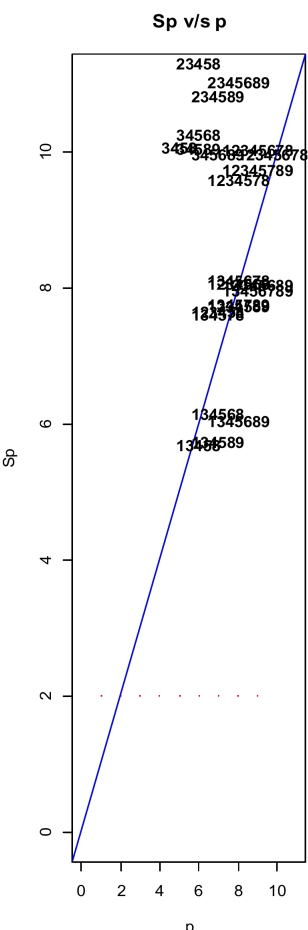

(c)

Figure 5. $C_{p}, R C_{p}$ and $S_{p}$ plots for Bayer Precipitation data: (a) $C_{p}$ versus $p$, (b) $R C_{p}$ versus $V_{p}$, (c) $S_{p}$ versus $p$.

For a real-life data set, it is difficult to show which submodel is good. Generally, the prediction error increases due to an addition of irrelevant variables in the model [34]. Consider three models suggested by [5] for further study, and compare the prediction error of these three models using GM-estimator and $\mathrm{k}$-fold cross-validation $(\mathrm{k}=10)$. The prediction error of $\left(X_{1}, X_{3}, X_{4}, X_{5}, X_{8}\right),\left(X_{1}, X_{3}, X_{4}, X_{5}, X_{7}, X_{8}\right)$ and $\left(X_{1}, X_{3}, X_{4}, X_{5}, X_{7}, X_{8}, X_{9}\right)$ are 
$57.8597,69.4523$ and 67.7013 respectively. The k-fold cross-validation study indicates that the model selected by $A S_{p}$ has a small prediction error as compared to other, and the prediction error of the model increases due to an addition of $X_{7}$ or $X_{7}$ and $X_{9}$. Thus, a subset of predictor variables namely $X_{1}, X_{3}, X_{4}, X_{5}, X_{8}$ is appropriate for fitting the model.

Table 4. $A S_{p}$ values and selected variables corresponding to different penalties.

\begin{tabular}{ccc}
\hline Penalty & Selected Variables & $A S_{p}$ (minimum) \\
\hline P1 & & 24.9337 \\
P2 & & 28.4348 \\
P3 & $X_{1}, X_{3}, X_{4}, X_{5}, X_{8}$ & 30.5247 \\
P4 & 36.5247 \\
P5 & & 56.2214 \\
P6 & & 49.7823 \\
\hline
\end{tabular}

\subsection{The performance of $A S_{p}$ criterion for different $\psi(\cdot)$ functions}

We have compared the performance of the $A S_{p}$ criterion based on GM-estimator and M-estimator with different $\psi(\cdot)$ functions in this section. The data was generated using the model and procedure explained in Section 2. The performance of three types of $\psi(\cdot)$ functions [15] i) Monotonic- Huber, Fair, ii) Soft Redescending- Cauchy, Welsch and iii) Hard Redescending- Tukey's bisquare (or biweight), Talwar has examined. The percentages of optimal model selection are calculated using 1000 simulated datasets, and the results are recorded in Tables 5-6. It is observed that, the hard and soft rescending functions are working well as compared to the monotonic functions. The performance of the M-estimator with redescending function is notable, and among redesceding functions, the Tukey's bisquare function has better performance. Nevertheless, the $A S_{p}$ criterion based on the GM-estimator selects the optimal model more precisely as compared to that based on the M-estimator. The performance is dependent on the choice of penalty, and the penalty function satisfying Conditions 3.4, 3.5 are selecting the optimal model with a high percentage. Overall, the criterion based on GM-estimator with the redescending function will be a better choice for small as well as large $n$.

\subsection{The performane of $A S_{p}$ criterion for difference $\omega(\cdot)$ weight functions}

In the previous simulation study, the weight function $\omega\left(X_{i}\right)=\sqrt{1-h_{i i}}$ based on the hat matrix has considered to compute the $A S_{p}$ criterion value. The hat matrix is hampered by a masking effect, and consequently, the diagonal values of the hat matrix do not always detect leverage points [30]. To overcome this problem, we can use alternative $\omega(\cdot)$ weights based on robust measures. The Robust Mahalnobis Distance (RMD) based on Minimum Volume Ellipsoid (MVE) can be used to identify leverage points $[11,29,30]$. The Generalized Potential (GP) is an another measure used to identify the leverage point, and the observation is said to be a high leverage point if corresponding GP value is greater than the threshold value Median $\left(G P_{i}\right)+3 M A D\left(G P_{i}\right)[1]$.

In this section, the superiority of the $A S_{p}$ criterion based on GM-estimator with different $\omega(\cdot)$ weights (Table 7 ) and $\psi(\cdot)$ functions is checked for severe cases: $5 \%$ vertical outliers and $5 \%$ leverage points, $10 \%$ vertical outliers and $10 \%$ leverage points, $15 \%$ vertical outliers and $15 \%$ leverage points. The data was generated using the model explained in the Section 2. Vertical outliers were included in the data multiplying by three to the response variable corresponding to maximum absolute residual, and bad leverage points were included in the data multiplying by three to response variable and row of the matrix X (excluding 1's column) corresponding to maximum leverage $\left(h_{i i}\right)$. The results are reported in the Table 8. A weight function $\omega_{1}$ is unable to detect all leverage points for large $n$ and consequently, 
it effect on the performance of the $A S_{p}$ criterion in presence of multiple leverage points. It is observed that, $A S_{p}$ criterion based on GM-estimator with robust weights $\omega_{3}, \omega_{4}$ and $\omega_{5}$ perform better in presence of multiple outliers. Also, the performance of $\omega_{2}$ is remarkable. The $A S_{p}$ criterion with hard rescending functions and robust weights perform well. Hence, the $A S_{p}$ criterion with hard rescending function with robust weight is a better choice in presence of multiple outliers.

Table 5. Performance of $A S_{p}$ criterion for GM-estimator and M-estimator with different $\psi(\cdot)$ functions.

\begin{tabular}{|c|c|c|c|c|c|c|c|c|c|c|c|c|c|c|}
\hline \multirow{2}{*}{$\mathrm{n}$} & \multirow{2}{*}{ Estimator } & \multirow{2}{*}{$\begin{array}{c}\psi(\cdot) \\
\text { function }\end{array}$} & \multicolumn{6}{|c|}{ 1-Vertical outlier and 1-Leverage point } & \multicolumn{6}{|c|}{ 2-Vertical outliers and 1-Leverage point } \\
\hline & & & P1 & P2 & P3 & $\mathrm{P} 4$ & P5 & P6 & P1 & P2 & P3 & P4 & $\mathrm{P} 5$ & P6 \\
\hline \multirow{12}{*}{50} & \multirow{6}{*}{$\begin{array}{c}\text { GM } \\
\text { estimator }\end{array}$} & Huber & 52.90 & 64.30 & 60.80 & 66.90 & 80.40 & 75.80 & 49.30 & 62.40 & 58.10 & 65.10 & 77.10 & 73.80 \\
\hline & & Fair & 43.00 & 53.80 & 49.70 & 55.60 & 70.40 & 65.00 & 40.10 & 51.80 & 46.80 & 53.80 & 66.90 & 63.50 \\
\hline & & Cauchy & 59.60 & 71.10 & 66.70 & 73.30 & 84.50 & 81.90 & 55.80 & 68.80 & 64.10 & 71.00 & 83.20 & 79.40 \\
\hline & & Welsch & 61.30 & 74.10 & 69.30 & 76.20 & 86.60 & 83.50 & 59.30 & 70.00 & 66.10 & 72.30 & 84.20 & 81.30 \\
\hline & & Bisquare & 61.50 & 74.00 & 69.50 & 76.50 & 86.90 & 83.50 & 59.30 & 69.70 & 66.40 & 72.50 & 84.50 & 81.20 \\
\hline & & Talwar & 60.00 & 71.80 & 67.60 & 73.70 & 83.10 & 80.70 & 58.50 & 69.10 & 65.90 & 71.20 & 82.80 & 80.40 \\
\hline & \multirow{6}{*}{$\begin{array}{c}\mathrm{M} \\
\text { estimator }\end{array}$} & Huber & 29.40 & 38.80 & 36.40 & 41.00 & 50.60 & 47.60 & 29.20 & 38.20 & 35.40 & 40.20 & 49.40 & 46.20 \\
\hline & & Fair & 23.30 & 31.70 & 28.10 & 32.90 & 41.60 & 40.10 & 22.30 & 31.00 & 28.50 & 32.90 & 42.60 & 40.00 \\
\hline & & Cauchy & 38.60 & 47.80 & 44.70 & 50.70 & 60.50 & 57.70 & 36.60 & 48.00 & 44.20 & 50.00 & 61.20 & 57.60 \\
\hline & & Welsch & 44.80 & 54.70 & 52.00 & 56.60 & 65.60 & 63.60 & 43.00 & 53.40 & 50.20 & 55.40 & 65.70 & 63.00 \\
\hline & & Bisquare & 43.60 & 54.40 & 51.30 & 56.30 & 65.20 & 63.00 & 43.20 & 53.60 & 49.90 & 55.50 & 65.50 & 63.00 \\
\hline & & Talwar & 30.20 & 37.60 & 35.20 & 39.00 & 45.30 & 44.10 & 29.40 & 37.50 & 34.10 & 38.70 & 45.70 & 43.90 \\
\hline \multirow{12}{*}{70} & \multirow{6}{*}{$\begin{array}{c}\text { GM } \\
\text { estimator }\end{array}$} & Huber & 60.10 & 71.90 & 70.40 & 75.60 & 86.70 & 85.90 & 56.00 & 69.70 & 67.70 & 74.10 & 86.30 & 85.30 \\
\hline & & Fair & 48.50 & 61.10 & 59.30 & 64.90 & 78.20 & 77.60 & 47.50 & 58.80 & 57.50 & 62.30 & 77.80 & 76.70 \\
\hline & & Cauchy & 64.60 & 77.10 & 75.60 & 81.50 & 89.80 & 89.50 & 60.70 & 74.20 & 72.50 & 78.50 & 89.50 & 88.60 \\
\hline & & Welsch & 66.80 & 79.50 & 78.10 & 83.60 & 91.40 & 90.90 & 62.50 & 76.30 & 75.00 & 80.70 & 92.00 & 91.30 \\
\hline & & Bisquare & 66.60 & 79.10 & 77.90 & 83.70 & 91.50 & 91.20 & 63.40 & 76.60 & 75.40 & 81.10 & 92.00 & 91.60 \\
\hline & & Talwar & 66.40 & 77.80 & 76.60 & 81.30 & 89.50 & 89.30 & 63.70 & 77.70 & 75.80 & 81.40 & 90.70 & 90.50 \\
\hline & \multirow{6}{*}{$\begin{array}{c}\mathrm{M} \\
\text { estimator }\end{array}$} & Huber & 38.10 & 49.60 & 47.90 & 53.10 & 64.60 & 64.50 & 38.00 & 49.20 & 46.70 & 52.70 & 65.30 & 63.80 \\
\hline & & Fair & 30.90 & 40.60 & 39.60 & 44.30 & 54.80 & 53.70 & 31.20 & 40.00 & 39.00 & 44.50 & 55.40 & 54.90 \\
\hline & & Cauchy & 50.60 & 62.70 & 61.40 & 67.30 & 78.00 & 77.30 & 47.40 & 60.00 & 57.70 & 64.50 & 76.90 & 75.90 \\
\hline & & Welsch & 57.30 & 70.30 & 68.10 & 74.00 & 84.80 & 84.40 & 53.90 & 65.90 & 63.80 & 70.60 & 82.40 & 81.40 \\
\hline & & Bisquare & 57.40 & 70.30 & 68.30 & 74.40 & 85.00 & 84.50 & 54.80 & 66.80 & 64.40 & 71.10 & 82.70 & 81.70 \\
\hline & & Talwar & 47.00 & 57.40 & 56.00 & 60.80 & 68.30 & 67.90 & 46.30 & 56.40 & 54.60 & 59.60 & 69.10 & 68.80 \\
\hline & & Huber & 65.00 & 75.50 & 75.90 & 80.30 & 91.20 & 92.50 & 63.50 & 73.50 & 74.00 & 79.20 & 91.90 & 92.80 \\
\hline & & Fair & 54.70 & 66.60 & 67.40 & 73.60 & 86.30 & 87.50 & 52.20 & 63.90 & 64.90 & 70.60 & 84.90 & 87.00 \\
\hline & GM & Cauchy & 68.90 & 79.50 & 80.20 & 84.20 & 94.00 & 95.30 & 66.60 & 79.40 & 80.30 & 85.20 & 95.10 & 95.80 \\
\hline & estimator & Welsch & 70.70 & 82.20 & 82.60 & 86.80 & 95.30 & 96.90 & 69.50 & 80.90 & 81.50 & 86.50 & 95.80 & 96.50 \\
\hline & & Bisquare & 71.20 & 82.70 & 82.90 & 87.00 & 95.20 & 96.80 & 69.80 & 81.30 & 82.10 & 87.30 & 95.90 & 96.50 \\
\hline 100 & & Talwar & 71.30 & 81.60 & 82.40 & 86.70 & 95.90 & 96.50 & 69.90 & 81.30 & 81.80 & 87.60 & 95.60 & 96.50 \\
\hline 100 & & Huber & 49.40 & 60.30 & 60.70 & 65.60 & 79.00 & 81.50 & 49.50 & 59.80 & 59.90 & 64.30 & 78.90 & 81.10 \\
\hline & & Fair & 40.10 & 50.10 & 50.40 & 54.80 & 68.40 & 70.70 & 37.90 & 48.40 & 48.60 & 53.40 & 68.50 & 70.50 \\
\hline & & Cauchy & 58.80 & 69.40 & 70.10 & 76.30 & 87.40 & 89.20 & 58.10 & 70.20 & 70.50 & 75.20 & 86.70 & 88.70 \\
\hline & estimator & Welsch & 65.60 & 77.20 & 77.70 & 82.70 & 92.30 & 93.60 & 63.30 & 75.80 & 76.30 & 81.30 & 91.80 & 93.60 \\
\hline & & Bisquare & 65.30 & 77.00 & 77.80 & 83.30 & 92.30 & 93.80 & 64.50 & 76.20 & 77.00 & 82.30 & 92.20 & 93.40 \\
\hline & & Talwar & 61.10 & 72.00 & 72.60 & 77.00 & 86.80 & 87.80 & 61.70 & 72.40 & 72.70 & 78.30 & 87.00 & 88.30 \\
\hline & & Huber & 68.70 & 81.40 & 85.10 & 89.30 & 95.90 & 98.40 & 67.20 & 81.20 & 85.20 & 88.90 & 96.70 & 98.60 \\
\hline & & Fair & 59.80 & 73.40 & 79.60 & 84.40 & 92.90 & 97.40 & 60.20 & 72.80 & 77.80 & 83.10 & 92.80 & 98.20 \\
\hline & GM & Cauchy & 72.80 & 85.10 & 89.30 & 92.30 & 97.30 & 99.50 & 72.60 & 84.90 & 88.40 & 91.50 & 97.60 & 99.50 \\
\hline & estimator & Welsch & 74.40 & 87.00 & 89.70 & 92.40 & 98.30 & 99.60 & 74.90 & 86.50 & 90.10 & 94.10 & 98.20 & 99.70 \\
\hline & & Bisquare & 74.40 & 87.20 & 89.70 & 92.40 & 98.30 & 99.60 & 75.00 & 86.60 & 90.40 & 94.10 & 98.30 & 99.60 \\
\hline 200 & & Talwar & 74.60 & 87.50 & 90.20 & 93.30 & 97.80 & 99.50 & 74.70 & 87.40 & 90.60 & 93.80 & 98.40 & 99.70 \\
\hline 200 & & Huber & 61.40 & 74.70 & 79.20 & 84.70 & 93.20 & 97.20 & 61.10 & 74.00 & 79.10 & 83.30 & 93.20 & 97.70 \\
\hline & & Fair & 50.40 & 64.90 & 69.60 & 75.80 & 87.40 & 93.30 & 50.20 & 64.90 & 69.30 & 74.20 & 86.40 & 92.50 \\
\hline & & Cauchy & 68.50 & 81.10 & 86.00 & 89.70 & 95.60 & 98.50 & 68.50 & 81.40 & 85.10 & 88.30 & 96.60 & 99.00 \\
\hline & estimator & Welsch & 71.10 & 85.60 & 89.10 & 91.60 & 97.60 & 99.20 & 71.90 & 84.60 & 88.30 & 92.20 & 98.00 & 99.60 \\
\hline & & Bisquare & 71.90 & 86.10 & 89.10 & 91.90 & 97.70 & 99.20 & 73.10 & 85.40 & 89.20 & 92.90 & 98.00 & 99.60 \\
\hline & & Talwar & 71.60 & 85.00 & 88.70 & 91.90 & 97.50 & 99.10 & 73.00 & 85.10 & 87.90 & 91.60 & 97.60 & 99.20 \\
\hline & & Huber & 73.60 & 85.40 & 90.60 & 93.10 & 98.20 & 100.00 & 72.70 & 85.40 & 91.20 & 93.60 & 98.40 & 99.80 \\
\hline & & Fair & 66.90 & 80.90 & 87.00 & 89.70 & 96.40 & 99.90 & 67.60 & 80.10 & 86.30 & 89.60 & 96.80 & 99.50 \\
\hline & GM & Cauchy & 76.20 & 89.60 & 93.30 & 95.10 & 98.60 & 99.90 & 77.00 & 88.80 & 92.30 & 95.90 & 98.90 & 100.00 \\
\hline & estimator & Welsch & 78.20 & 89.60 & 94.10 & 95.90 & 98.70 & 99.90 & 77.90 & 89.20 & 93.90 & 96.60 & 99.00 & 100.00 \\
\hline & & Bisquare & 78.30 & 89.70 & 94.20 & 95.90 & 98.80 & 99.90 & 77.50 & 89.40 & 93.60 & 96.70 & 99.00 & 100.00 \\
\hline & & Talwar & 78.90 & 89.70 & 94.20 & 96.00 & 98.90 & 99.70 & 78.00 & 88.30 & 93.30 & 96.30 & 99.00 & 100.00 \\
\hline 300 & & Huber & 69.00 & 81.20 & 88.00 & 90.50 & 97.00 & 99.90 & 69.20 & 82.20 & 87.60 & 91.90 & 97.00 & 99.50 \\
\hline & & Fair & 61.00 & 75.00 & 81.70 & 85.90 & 94.90 & 99.30 & 62.40 & 75.20 & 81.80 & 85.80 & 93.90 & 98.70 \\
\hline & & Cauchy & 73.90 & 87.20 & 92.00 & 94.30 & 98.20 & 99.80 & 74.40 & 87.30 & 91.30 & 94.70 & 98.40 & 99.70 \\
\hline & estimator & Welsch & 76.70 & 89.00 & 93.40 & 95.80 & 98.60 & 99.70 & 76.10 & 88.40 & 92.70 & 96.00 & 98.70 & 99.90 \\
\hline & & Bisquare & 77.30 & 89.10 & 93.90 & 95.80 & 98.60 & 99.70 & 76.40 & 88.20 & 92.70 & 96.40 & 98.90 & 100.00 \\
\hline & & Talwar & 77.30 & 88.80 & 93.30 & 95.30 & 98.50 & 99.70 & 76.70 & 87.30 & 92.50 & 95.90 & 99.10 & 100.00 \\
\hline
\end{tabular}


Table 6. Performance of $A S_{p}$ criterion for GM-estimator and M-estimator with different $\psi(\cdot)$ function.

\begin{tabular}{|c|c|c|c|c|c|c|c|c|c|c|c|c|c|c|}
\hline \multirow[b]{2}{*}{$\mathrm{n}$} & \multirow{2}{*}{ Estimator } & \multirow{2}{*}{$\begin{array}{c}\psi(\cdot) \\
\text { function }\end{array}$} & \multicolumn{6}{|c|}{ 1-Vertical outlier and 2-Leverage points } & \multicolumn{6}{|c|}{ 2-Vertical outliers and 2-Leverage points } \\
\hline & & & P1 & P2 & P3 & P4 & P5 & P6 & P1 & P2 & P3 & P4 & P5 & P6 \\
\hline \multirow{12}{*}{50} & \multirow{6}{*}{$\begin{array}{c}\text { GM } \\
\text { estimator }\end{array}$} & Huber & 30.90 & 41.40 & 37.40 & 44.10 & 58.50 & 53.60 & 31.70 & 42.10 & 38.60 & 44.70 & 59.70 & 55.70 \\
\hline & & Fair & 21.50 & 31.90 & 27.00 & 33.80 & 46.40 & 43.10 & 23.90 & 33.80 & 30.70 & 35.50 & 47.20 & 43.30 \\
\hline & & Cauchy & 40.80 & 53.00 & 48.20 & 55.30 & 68.10 & 63.40 & 39.50 & 51.80 & 47.50 & 53.20 & 66.60 & 63.60 \\
\hline & & Welsch & 47.30 & 58.90 & 55.00 & 61.00 & 72.90 & 69.20 & 46.50 & 58.10 & 54.00 & 60.00 & 71.90 & 67.90 \\
\hline & & Bisquare & 47.50 & 58.60 & 55.50 & 60.60 & 72.90 & 69.70 & 48.10 & 59.00 & 55.10 & 60.90 & 72.90 & 69.40 \\
\hline & & Talwar & 38.40 & 46.90 & 43.60 & 48.20 & 57.50 & 54.90 & 38.70 & 48.30 & 45.10 & 49.60 & 59.80 & 57.60 \\
\hline & \multirow{6}{*}{$\begin{array}{c}\mathrm{M} \\
\text { estimator }\end{array}$} & Huber & 13.70 & 19.20 & 16.60 & 20.10 & 29.70 & 28.00 & 15.50 & 20.80 & 18.90 & 21.70 & 29.40 & 27.70 \\
\hline & & Fair & 12.60 & 18.20 & 16.00 & 19.10 & 24.40 & 22.90 & 14.20 & 18.90 & 16.90 & 19.20 & 25.40 & 23.60 \\
\hline & & Cauchy & 18.10 & 24.10 & 21.70 & 25.80 & 35.20 & 33.00 & 17.20 & 23.50 & 21.10 & 24.90 & 34.50 & 30.90 \\
\hline & & Welsch & 24.10 & 31.40 & 28.30 & 33.10 & 41.30 & 38.60 & 23.50 & 30.90 & 28.70 & 32.80 & 41.00 & 38.90 \\
\hline & & Bisquare & 24.70 & 32.00 & 29.00 & 33.80 & 41.70 & 38.60 & 23.70 & 30.90 & 28.90 & 32.80 & 41.80 & 39.80 \\
\hline & & Talwar & 12.90 & 17.00 & 15.20 & 17.90 & 24.00 & 22.10 & 16.70 & 20.60 & 18.50 & 21.40 & 26.60 & 24.90 \\
\hline \multirow{12}{*}{70} & \multirow{6}{*}{$\begin{array}{c}\text { GM } \\
\text { estimator }\end{array}$} & Huber & 39.50 & 49.50 & 48.10 & 53.40 & 68.70 & 67.00 & 39.60 & 52.00 & 49.80 & 55.90 & 71.10 & 69.50 \\
\hline & & Fair & 29.10 & 39.40 & 38.00 & 42.70 & 56.50 & 55.90 & 30.70 & 40.50 & 38.90 & 44.70 & 57.40 & 56.60 \\
\hline & & Cauchy & 51.00 & 60.60 & 58.90 & 65.60 & 79.20 & 78.10 & 50.90 & 63.10 & 61.00 & 66.80 & 80.50 & 80.10 \\
\hline & & Welsch & 56.10 & 67.10 & 65.00 & 72.20 & 83.90 & 82.90 & 58.70 & 68.70 & 67.30 & 73.10 & 84.20 & 83.30 \\
\hline & & Bisquare & 56.30 & 67.50 & 65.90 & 72.10 & 83.70 & 83.20 & 59.10 & 69.70 & 67.90 & 73.60 & 85.30 & 84.00 \\
\hline & & Talwar & 50.40 & 60.30 & 58.40 & 64.30 & 76.20 & 75.80 & 53.60 & 64.40 & 62.50 & 67.80 & 79.50 & 78.70 \\
\hline & \multirow{6}{*}{$\begin{array}{c}\mathrm{M} \\
\text { estimator }\end{array}$} & Huber & 21.00 & 28.60 & 27.40 & 31.70 & 42.70 & 42.10 & 23.90 & 31.10 & 30.10 & 34.20 & 46.00 & 45.00 \\
\hline & & Fair & 15.40 & 22.80 & 21.20 & 24.50 & 33.40 & 33.00 & 19.10 & 25.90 & 25.20 & 27.40 & 36.10 & 35.30 \\
\hline & & Cauchy & 32.00 & 41.10 & 40.00 & 44.60 & 55.50 & 54.70 & 31.90 & 40.30 & 39.70 & 43.00 & 58.60 & 56.80 \\
\hline & & Welsch & 42.80 & 52.20 & 50.90 & 56.60 & 68.80 & 68.30 & 44.10 & 57.00 & 54.60 & 60.30 & 71.20 & 70.90 \\
\hline & & Bisqu & 43.40 & 52.10 & 50.90 & 56.80 & 68.40 & 68.10 & 44.40 & 56.60 & 54.70 & 59.90 & 71.20 & 70.90 \\
\hline & & Talwar & 26.70 & 33.40 & 32.20 & 36.00 & 43.30 & 43.00 & 29.90 & 36.10 & 34.80 & 38.80 & 47.50 & 47.00 \\
\hline & & Huber & 43.60 & 54.90 & 55.40 & 61.20 & 74.80 & 76.70 & 40.00 & 53.10 & 53.60 & 59.60 & 74.60 & 76.40 \\
\hline & & Fair & 32.80 & 44.60 & 45. & 50.60 & 64.30 & 66.80 & 31.80 & 42.70 & 43.10 & 48.10 & 62.00 & 63.90 \\
\hline & & Cauc & 52.10 & 65.10 & 66.30 & 72.90 & 85.90 & 87.90 & 49.90 & 64.80 & 65.00 & 72.30 & 85.30 & 86.80 \\
\hline & & Welsch & 59.40 & 73.10 & 73.80 & 78.80 & 90.30 & 91.50 & 57.50 & 70.80 & 71.60 & 77.10 & 89.40 & 90.90 \\
\hline & & Bisquare & 59.70 & 73.90 & 74.80 & 79.30 & 90.40 & 91.80 & 57.70 & 71.20 & 71.80 & 77.20 & 89.50 & 90.70 \\
\hline & & Talwar & 55.90 & 69.90 & 70.10 & 74.60 & 86.40 & 87.70 & 56.50 & 69.30 & 69.80 & 75.20 & 86.60 & 88.00 \\
\hline 100 & & Huber & 28.40 & 38.20 & 38.50 & 43.40 & 57.10 & 59.70 & 27.70 & 37.10 & 37.30 & 42.20 & 54.70 & 57.70 \\
\hline & & Fair & 21.60 & 29.10 & 29.30 & 33.70 & 46.20 & 49.90 & 20.30 & 27.20 & 27.70 & 33.00 & 45.20 & 47.50 \\
\hline & & Cauchy & 39.80 & 50.40 & 50.70 & 54.50 & 68.40 & 70.70 & 37.10 & 48.40 & 49.40 & 54.90 & 68.60 & 70.60 \\
\hline & estimator & & 49.40 & 63.80 & 64.8 & 71.00 & 83.00 & 84.50 & & 62.1 & & 68.40 & 81.50 & 83.90 \\
\hline & & Bisqu & 50.60 & 65.50 & 66.40 & 71.40 & 83.30 & 84.80 & 50.80 & 62.80 & 63.70 & 69.50 & 82.60 & 84.10 \\
\hline & & Talwar & 41.50 & 53.20 & 53.50 & 58.10 & 68.20 & 70.00 & 42.00 & 53.60 & 53.90 & 60.10 & 69.80 & 71.40 \\
\hline & & Huber & 55.30 & 68.50 & 72.70 & 77.20 & 88.30 & 94.10 & 56.90 & 67.60 & 72.60 & 77.20 & 89.70 & 94.80 \\
\hline & & & 43.10 & 57. & 60. & 65.80 & 78.50 & 86.10 & 45. & 57. & & 66.50 & 79.70 & 88.00 \\
\hline & & Cauchy & 65.10 & 79.60 & 84.00 & 87.00 & 95.50 & 97.30 & 66.20 & 78.80 & 82.90 & 87.00 & 95.10 & 98.40 \\
\hline & & Welsch & 70.10 & 82.50 & 87.30 & 91.50 & 96.80 & 98.10 & 71.00 & 83.00 & 87.90 & 90.50 & 96.50 & 99.30 \\
\hline & & Bisqu & 70.40 & 82. & & 91.40 & 97.10 & 98.2 & & & & 90.90 & 96.90 & 99.40 \\
\hline & & Tal & 69.30 & 81.60 & 86.80 & 91.00 & 96.50 & 97.90 & 71.90 & 83.90 & 87.60 & 90.80 & 96.60 & 98.60 \\
\hline 200 & & Huber & 44.90 & 58.10 & 63.10 & 67.60 & 80.40 & 88.40 & 47.50 & 60.80 & 64.10 & 68.40 & 82.00 & 88.80 \\
\hline & & & 34.00 & 45.10 & 49.80 & 55.00 & 68.70 & 77.50 & & 47.50 & 51.90 & 56.70 & 70.30 & 79.40 \\
\hline & & Cauchy & 57.80 & 72.30 & 77.40 & 81.50 & 91.00 & 95.50 & 59.80 & 71.90 & 75.60 & 80.80 & 90.50 & 95.80 \\
\hline & estimator & Welsch & 67.00 & 79.90 & 84.80 & 89.30 & 95.70 & 97.50 & 67.20 & 80.60 & 84.80 & 87.90 & 95.50 & 98.20 \\
\hline & & & 67.50 & & & 89.40 & 95.90 & 97.60 & & & & 89.30 & 96.10 & 98.60 \\
\hline & & Talwar & 65.50 & 78.90 & 83.80 & 87.90 & 94.60 & 96.80 & 68.90 & 80.70 & 84.50 & 87.70 & 93.60 & 96.80 \\
\hline & & Huber & 63.10 & 76.10 & 82.50 & 86.50 & 95.60 & 98.70 & 62.60 & 76.00 & 82.70 & 86.90 & 94.90 & 98.70 \\
\hline & & & 52.80 & 66.50 & 72.90 & 77.80 & 89.40 & 96.60 & 53.90 & 64.60 & 72.70 & 77.70 & 89.00 & 97.00 \\
\hline & & Cauchy & 70.20 & 83.60 & 90.10 & 93.00 & 97.60 & 99.40 & 72.10 & 84.70 & 90.10 & 92.90 & 97.80 & 99.70 \\
\hline & estimator & Welsch & 73.40 & 86.10 & 92.10 & 94.60 & 98.20 & 99.90 & 75.10 & 87.10 & 91.20 & 93.70 & 98.60 & 99.90 \\
\hline & & Bisquare & 73.70 & 86.70 & 92.20 & 94.50 & 98.50 & 99.90 & 74.90 & 87.70 & 91.30 & 94.00 & 98.90 & 99.90 \\
\hline & & Talwar & 74.30 & 86.90 & 91.50 & 94.80 & 98.40 & 99.80 & 75.60 & 86.80 & 92.00 & 94.10 & 98.70 & 99.90 \\
\hline 300 & & Huber & 57.00 & 70.00 & 77.50 & 82.10 & 91.90 & 97.40 & 57.80 & 68.60 & 77.30 & 81.40 & 91.60 & 98.00 \\
\hline & & Fair & & 58.90 & 66.90 & 71.10 & 83.10 & 93.70 & 46.70 & 58.40 & 65.40 & 69.90 & 83.40 & 92.70 \\
\hline & & Cauchy & 66.20 & 80.30 & 86.50 & 90.00 & 96.60 & 98.80 & 66.90 & 79.60 & 86.40 & 90.80 & 96.70 & 98.90 \\
\hline & estimator & Welsch & 71.40 & 84.90 & 91.40 & 93.40 & 98.00 & 99.70 & 73.20 & 86.00 & 90.30 & 93.00 & 98.20 & 99.70 \\
\hline & & Bisquare & 72.30 & 84.80 & 91.60 & 93.40 & 98.10 & 99.80 & 73.50 & 86.30 & 90.30 & 93.10 & 98.40 & 99.70 \\
\hline & & Talwar & 72.40 & 85.20 & 90.50 & 93.60 & 98.10 & 99.50 & 73.70 & 85.30 & 91.00 & 93.10 & 98.50 & 99.80 \\
\hline
\end{tabular}

Table 7. List of $\omega(\cdot)$ weight functions.

\begin{tabular}{cc}
\hline Sr. No. & \multicolumn{1}{c}{$\omega(\cdot)$ weight function } \\
\hline 1. & $\omega_{1}\left(X_{i}\right)=\sqrt{1-h_{i i}}$ \\
\hline 2. & $\omega_{2}\left(X_{i}\right)= \begin{cases}1, & \text { if } h_{i i} \leq \frac{2 p}{n} \\
0, & \text { otherwise }\end{cases}$ \\
\hline 3. & $\omega_{3}\left(X_{i}\right)= \begin{cases}1, & \text { if } R M D_{i} \leq \text { Median }\left(R M D_{i}\right)+3 M A D\left(R M D_{i}\right) \\
0, & \text { otherwise }\end{cases}$ \\
\hline 4. & $\omega_{4}\left(X_{i}\right)= \begin{cases}1, & \text { if } R M D_{i} \leq \sqrt{\chi_{p, 0.95}^{2}} \\
0, & \text { otherwise }\end{cases}$ \\
\hline 5. & $\omega_{5}\left(X_{i}\right)= \begin{cases}1, & \text { if } G P_{i} \leq \text { Median }\left(G P_{i}\right)+3 M A D\left(G P_{i}\right) \\
0, & \text { otherwise }\end{cases}$ \\
\hline
\end{tabular}


Table 8. Performance of $A S_{p}$ criterion for different $\omega(\cdot)$ weight functions.

\begin{tabular}{|c|c|c|c|c|c|c|}
\hline & & $\psi(\cdot)$ & $5 \%$-Ve & rtical & thliers a & $105 \%-1$ \\
\hline & weight & function & P1 & P2 & P3 & $\mathrm{P} 4$ \\
\hline & & Huber & 8.40 & 11.50 & 11.70 & 14.10 \\
\hline & & Fair & 6.80 & 10.10 & 10.60 & 11.70 \\
\hline & & Cauchy & 16.00 & 22.60 & 23.00 & 27.00 \\
\hline & & Welsch & 37.50 & 47.50 & 47.80 & 53.50 \\
\hline & & Bisquare & 38.30 & 48.70 & 48.80 & 54.60 \\
\hline & & Talwar & 22.00 & 28.90 & 29.30 & 32.10 \\
\hline & & Huber & 44.50 & 57.40 & 57.70 & 63.60 \\
\hline & & Fair & 38.40 & 50.50 & 51.00 & 56.00 \\
\hline & & Cauchy & 47.80 & 58.80 & 58.90 & 66.10 \\
\hline & & Welsch & 49.40 & 61.00 & 61.50 & 67.50 \\
\hline & & Bisquare & 50.00 & 61.50 & 61.60 & 67.80 \\
\hline & & Talwar & 50.50 & 62.60 & 63.30 & 68.80 \\
\hline & & Huber & 46.50 & 57.40 & 58.00 & 63.80 \\
\hline & & Fair & 39.30 & 52.00 & 52.40 & 57.00 \\
\hline & & Cauchy & 47.90 & 59.10 & 59.00 & 65.90 \\
\hline 100 & & Welsch & 49.20 & 60.70 & 61.30 & 67.50 \\
\hline & & Bisquare & 49.70 & 61.10 & 61.30 & 67.80 \\
\hline & & Talwar & 50.50 & 62.00 & 62.70 & 68.00 \\
\hline & & Huber & 44.50 & 55.80 & 56.70 & 62.70 \\
\hline & & Fair & 38.10 & 51.10 & 51.80 & 56.30 \\
\hline & & Cauchy & 47.60 & 58.90 & 58.90 & 64.50 \\
\hline & & Welsch & 49.70 & 60.30 & 60.60 & 66.70 \\
\hline & & Bisquare & 49.80 & 60.20 & 60.80 & 67.40 \\
\hline & & Talwar & 49.90 & 62.00 & 62.80 & 68.20 \\
\hline & & Huber & 45.20 & 56.70 & 57.10 & 62.90 \\
\hline & & Fair & 38.50 & 49.50 & 49.90 & 56.00 \\
\hline & & Cauchy & 47.90 & 58.60 & 58.90 & 65.70 \\
\hline & & Welsch & 49.40 & 60.60 & 61.30 & 66.60 \\
\hline & & Bisqu & 49.70 & 60.90 & 61.20 & 66.80 \\
\hline & & Talwar & 50.60 & 62.00 & 62.80 & 68.00 \\
\hline & & Huber & 1.20 & 2.30 & 2.80 & 4.40 \\
\hline & & Fair & 1.20 & 1.7 & 2.1 & 2.90 \\
\hline & & Cauchy & 5.50 & 8.20 & 10.20 & 11.70 \\
\hline & $\omega_{1}(X$ & Welsch & 40.50 & 50.20 & 54.50 & 8.50 \\
\hline & & Bisq & 43.10 & 53.30 & 58.00 & 63.30 \\
\hline & & Talwar & 27.70 & 34.80 & 38.80 & 41.50 \\
\hline & & uber & 43.60 & 56.60 & 62.60 & 68.80 \\
\hline & & $\mathrm{Fa}$ & 37.00 & 48.70 & 55.00 & 0.40 \\
\hline & & Cauchy & 47.70 & 59.70 & 64.70 & 0.50 \\
\hline & & Welsch & 49.90 & 61.80 & 67.00 & 72.20 \\
\hline & & Bisqu & 49.70 & 62.00 & 67.30 & 72.30 \\
\hline & & Talwar & 50.10 & 62.90 & 68.30 & 73.40 \\
\hline & & uber & 43.30 & 57.40 & 63.20 & 8.50 \\
\hline & & Fair & 36.80 & 49.40 & 55.20 & 60.50 \\
\hline & & Cauchy & 47.50 & 59.90 & 64.50 & 70.50 \\
\hline 200 & & Wels & 49.90 & 61.80 & 67.00 & 72.20 \\
\hline & & Bisq & 49.60 & 62.00 & 67.30 & 72.20 \\
\hline & & Talwar & 50.30 & 62.90 & 68.30 & 73.40 \\
\hline & & & 43.4 & 57.80 & 62.7 & 68 \\
\hline & & $\mathrm{Fa}$ & 37.20 & 49.60 & 55.50 & 0.40 \\
\hline & & Cauc & 46.60 & 59.80 & 64.60 & .60 \\
\hline & & Wels & 49.50 & 61.60 & 67.00 & 71.70 \\
\hline & & Bisqu & 49.10 & 62.00 & 67.10 & 72.00 \\
\hline & & & 49.80 & 63.10 & 68.40 & 10 \\
\hline & & & 43.20 & 57.30 & 62.6 & 0 \\
\hline & & Fair & 37.10 & 49.40 & 55.10 & 9.70 \\
\hline & & Cauchy & 47.10 & 59.70 & 64.30 & 70 \\
\hline & & Wels & 49.60 & 61.80 & 66.70 & 71.90 \\
\hline & & Bisqu & 49.30 & 62.10 & 67.00 & 72.10 \\
\hline & & & 50.00 & 62.80 & 68.2 & \\
\hline & & & 0.30 & 0.4 & 0.60 & \\
\hline & & & 0.0 & 0.2 & 0.2 & \\
\hline & & & 1.2 & 2.50 & 2.90 & 3.3 \\
\hline & & Wels & 34.30 & 44.90 & 52.20 & 56.60 \\
\hline & & Bisq & 8.90 & 52.10 & 58.60 & 1.00 \\
\hline & & & 29.5 & 38.40 & 43.3 & 47. \\
\hline & & $\mathrm{H}$ & & 56 & 4.6 & 0 \\
\hline & & & 31.50 & 16.70 & 56.00 & .50 \\
\hline & & & 44.50 & 58.60 & 66.00 & 71.70 \\
\hline & & Wels & 46.50 & 1.20 & 67.90 & 10 \\
\hline & & Bisq & 80 & 1.60 & & .50 \\
\hline & & & & 2.50 & & \\
\hline & & & 4 & 5 & 0 & 6 \\
\hline & & & 0.90 & 47.10 & 10 & 40 \\
\hline & & & 44.40 & 58.80 & 65.8 & 71.50 \\
\hline 300 & & & 3.50 & 61.30 & 0 & 7 \\
\hline & & Bisqu & 6.80 & 61.60 & & .50 \\
\hline & & & & & & \\
\hline & & & & j & & \\
\hline & & & 0.90 & 7.30 & & \\
\hline & & & & & & \\
\hline & & & & 0 & & 72 \\
\hline & & Bisqu & 6.90 & 1.70 & 68.00 & 73.5 \\
\hline & & & & 25 & & \\
\hline & & & & 55.40 & 0 & 6 \\
\hline & & & 31.0 & 46.70 & & \\
\hline & & & & & & \\
\hline & & & & 0 & & \\
\hline & & Bisquare & 6.70 & 61.70 & 67.90 & 73.60 \\
\hline & & Talwar & 48.70 & 62.60 & 68.90 & 74.80 \\
\hline
\end{tabular}




\section{Discussion}

We have suggested the $A S_{p}$ model selection criterion based on GM-estimator. It can be viewed as a generalization of $C_{p}$ and $S_{p}$ criteria. The proposed criterion takes into account a discrepancy between the full model and submodel as well as complexity in the model. This criterion has consistency property and selects an optimal model with a high probability for large $n$. The simulation study reveals that the proposed criterion works well in the four different situations: clean data, vertical outliers, leverage points, and both vertical outliers and leverage points. The proposed criterion performs well and simple as compared to $C_{p}, S_{p}$ and $R C_{p}$. The $A S_{p}$ criterion based on the GM-estimator with hard redescending $\psi(\cdot)$ function and robust weight can be a better choice.

\section{Acknowledgments}

We sincerely thank the Editor, Associate Editor and anonymous reviewers for their careful review, constructive comments and valuable suggestions that resulted in the significant improvement of the original manuscript. We would also like to thanks DST, New Delhi and UGC, New Delhi for financial support under DST-FIST and UGC-SAP scheme, respectively.

\section{References}

[1] M. Alguraibawi, H. Midi and A.H.M. Imon, A new robust diagnostic plot for classifying good and bad high leverage points in a multiple linear regression model, Math. Probl. Eng., Doi:10.1155/2015/279472, 2015.

[2] C.D.S. André, S.N. Elian, S.C. Narula and R.A. Tavares, Coefficients of determinations for variable selection in the msae regression, Comm. Statist. Theory Methods 29 (3), 623-642, 2000.

[3] B. Asikgil and A. Erar, Research into multiple outliers in linear regression analysis, Hacet. J. Math. Stat. 38 (2), 185-198, 2009.

[4] A.C. Atkinson, Fast very robust methods for the detection of multiple outliers, J. Amer. Statist. Assoc. 89 (428), 1329-1339, 1994.

[5] A. Bab-Hadiashar and D. Suter, Data Segmentation and Model Selection for Computer Vision: A Statistical Approach, Springer, 2000.

[6] D.A. Belsley, E. Kuh and R.E. Welsch, Regression Diagnostics: Identifying Influential Data and Sources of Collinearity, Wiley, 1980.

[7] H. Bozdogan and D.M.A. Haughton, Informational complexity criteria for regression models, Comput. Statist. Data Anal. 28 (1), 51-76, 1998.

[8] S. Chatterjee and A.S. Hadi, Influential observations, high leverage points and outliers in linear regression, Statist. Sci. 1 (3), 379-416, 1986.

[9] C.W. Coakley and T.P. Hettmansperger, A bounded influence, high breakdown, efficient regression estimator, J. Amer. Statist. Assoc. 88 (423), 872-880, 1993.

[10] C. Croux and C. Dehon, Estimators of the multiple correlation coefficient: Local robustness and confidence intervals, Statist. Papers 44 (3), 315-334, 2003.

[11] C. Dehon, M. Gassner and V. Verardi, Beware of 'Good' outliers and overoptimistic conclusions, Oxf. Bull. Econ. Stat. 71 (3), 437-452, 2009.

[12] A.S. Hadi and J.S. Simonoff, Procedures for the identification of multiple outliers in linear models, J. Amer. Statist. Assoc. 88 (424), 1264-1272, 1993.

[13] F. R. Hampel, E.M. Ronchetti, P.J. Rousseeuw and W.A. Stahel, Robust Statistics: The Approach based on Influence Functions, Wiley, 1986.

[14] R.W. Hill, When there are outliers in the carriers: The univariate case, Comm. Statist. Theory Methods 11 (8), 849-868, 1982.

[15] P.W. Holland and R.E. Welsch, Robust regression using iteratively reweighted leastsquares, Comm. Statist. Theory Methods 6 (9), 813-827, 1977. 
[16] B. Hu and J. Shao, Generalized linear model selection using $R^{2}$, J. Statist. Plann. Inference 138 (12), 3705-3712, 2008.

[17] P.J. Huber, Robust estimation of a location parameter, Ann. Math. Stat. 35 (1), 73-101, 1964.

[18] D.N. Kashid and S.R. Kulkarni, A more general criterion for subset selection in multiple linear regression, Comm. Statist. Theory Methods 31 (5), 795-811, 2002.

[19] C. Kim and S. Hwang, Influence subsets on the variable selection, Comm. Statist. Theory Methods 29 (2), 335-347, 2000.

[20] W.S. Krasker and R.E. Welsch, Efficient bounded-influence regression estimation, J. Amer. Statist. Assoc. 77 (379), 595-604, 1982.

[21] J.A.F. Machado, Robust Model Selection and M-estimation, Econometric Theory 9 (3), 478-493, 1993.

[22] C. Mallows, Some comments on $C_{p}$, Technometrics 15 (4), 661-675, 1973.

[23] R.A. Maronna, R.D. Martin and V.J. Yohai, Robust Statistics: Theory and Methods, Wiley, 2006.

[24] R.A. Maronna and V.J. Yohai, Asymptotic behavior of general M-estimates for regression and scale with random carriers, Probab. Theory Related Fields 58 (1), 7-20, 1981.

[25] C.R. Rao, Y. Wu, S. Konishi and R. Mukerjee, On model selection, Lecture Notes in Monograph Series 38, 1-64, 2001.

[26] O. Renaud and M.P. Victoria-Feser, A robust coefficient of determination for regression, J. Statist. Plann. Inference 140 (7), 1852-1862, 2010.

[27] E. Ronchetti, Robust model selection in regression, Statist. Probab. Lett. 3 (1), 21-23, 1985.

[28] E. Ronchetti and R.G. Staudte, A robust version of Mallows's $C_{p}$, J. Amer. Statist. Assoc. 89 (426), 550-559, 1994.

[29] P.J. Rousseeuw and A.M. Leroy, Robust Regression and Outlier Detection, Wiley, 2003.

[30] P.J. Rousseeuw and B.C. Van Zomeren, Unmasking multivariate outliers and leverage points, J. Amer. Statist. Assoc. 85 (411), 633-639, 1990.

[31] S. Sommer and R.G. Staudte, Robust variable selection in regression in the presence of outliers and leverage points, Aust. N. Z. J. Stat. 37 (3), 323-336, 1995.

[32] K. Tharmaratnam and G. Claeskens, A comparison of robust versions of the AIC based on M, S and MM-estimators, Statistics 47 (1), 216-235, 2013.

[33] R. Wilcox, Introduction to Robust Estimation and Hypothesis Testing, Elsevier, 2012.

[34] R. Wilcox, Modern Statistics for the Social and Behavioral Sciences: A Practical Introduction, CRC Press, 2012. 